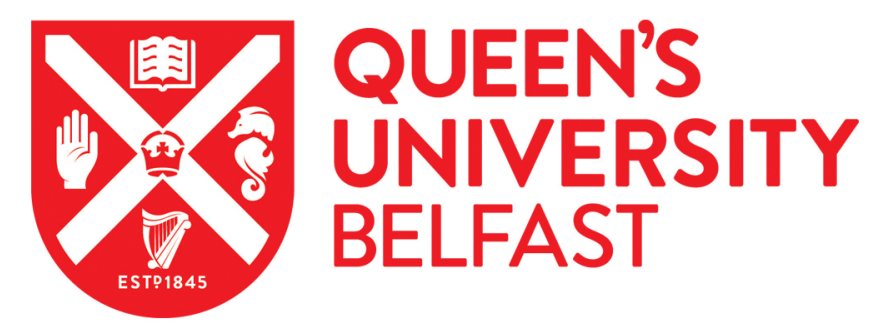

\title{
Improving the crystallinity and magnetocaloric effect of the perovskite La0.65Sr0.35MnO3 using microwave irradiation
}

McBride, K., Bennington-Gray, S., Cook, J., Stella, L., Felton, S., \& Poulidi, D. (2017). Improving the crystallinity and magnetocaloric effect of the perovskite La0.65Sr0.35MnO3 using microwave irradiation. CrystEngComm, 19, 3776-3791. https://doi.org/10.1039/C7CE00882A

\section{Published in:}

CrystEngComm

\section{Document Version:}

Peer reviewed version

Queen's University Belfast - Research Portal:

Link to publication record in Queen's University Belfast Research Portal

\section{Publisher rights}

(C) The Royal Society of Chemistry 2017

This work is made available online in accordance with the publisher's policies. Please refer to any applicable terms of use of the publisher.

\section{General rights}

Copyright for the publications made accessible via the Queen's University Belfast Research Portal is retained by the author(s) and / or other copyright owners and it is a condition of accessing these publications that users recognise and abide by the legal requirements associated with these rights.

Take down policy

The Research Portal is Queen's institutional repository that provides access to Queen's research output. Every effort has been made to ensure that content in the Research Portal does not infringe any person's rights, or applicable UK laws. If you discover content in the Research Portal that you believe breaches copyright or violates any law, please contact openaccess@qub.ac.uk. 


\section{CrystEngComm}

\section{ARTICLE}

\section{Improving the crystallinity and magnetocaloric effect of the perovskite $\mathrm{La}_{0.65} \mathrm{Sr}_{0.35} \mathrm{MnO}_{3}$ using microwave irradiation}

Received 00th January 20xx, Accepted 00th January 20xx DOI: $10.1039 / x 0 x \times 00000 x$ www.rsc.org/

\author{
Katherine McBride ${ }^{1}$, Suzanne Bennington-Gray ${ }^{2}$, James Cook ${ }^{1,2}$, Lorenzo Stella ${ }^{1,2}$ Solveig Felton ${ }^{2}$, \\ and Danai Poulidi ${ }^{1 *}$
}

The use of microwave (MW) irradiation to assist the modified peroxide sol-gel synthesis of $\mathrm{La}_{1-\mathrm{x}} \mathrm{Sr}_{\mathrm{x}} \mathrm{MnO}_{3}$ was shown to 1)improve crystallinity and 2)enhance the magnetic susceptibility and magnetocaloric effect (MCE) of samples compared to those synthesised using the conventional method. The improvement in crystallinity of samples synthesised using MW irradiation was evident in the structural characterisation conducted using SEM and XRD. Magnetic heating experiments on $\mathrm{La}_{1-\mathrm{x}} \mathrm{Sr}_{\mathrm{x}} \mathrm{MnO}_{3}(\mathrm{x}=0.25,0.35$ and 0.4$)$ showed that for the MW-assisted method, the most suitable material for magnetic fluid hyperthermia applications was $\mathrm{La}_{0.65} \mathrm{Sr}_{0.35} \mathrm{MnO}_{3}$. MW settings (time and power) were investigated in an attempt to further optimise the MCE. Increasing MW time settings used (10 min, 30 min and 2 hours) led to increased saturation temperatures $\left(45.9^{\circ} \mathrm{C}, 57.0^{\circ} \mathrm{C}\right.$ and $58.3^{\circ} \mathrm{C}$ respectively), with a linear relationship between the MW power settings used and the MCE also observed during magnetic heating experiments. Specific absorption rates as high as $175 \mathrm{Wg}^{-1} \mathrm{Mn}$ were achieved compared to the conventional maximum of $56 \mathrm{Wg}^{-1} \mathrm{Mn}$ in previous work. The enhancement of MCE was ascribed to a greater core ferromagnetic contribution as a result of the improvement in the degree of crystallinity and magnetic susceptibility of the samples synthesised with MW-irradiation .

\section{Introduction}

Lanthanum strontium manganate, $\mathrm{La}_{1-x} \mathrm{Sr}_{x} \mathrm{MnO}_{3}(\mathrm{LSMO})$ is a material which has been heavily investigated for a variety of applications; from its use as an industrial oxidative catalyst ${ }^{1}$, a fuel-cell electrode ${ }^{2,3}$, to a mediator for Magnetic Fluid Hyperthermia (MFH), which this work is directed towards. ${ }^{4-8}$ The therapeutic use of heat between $41{ }^{\circ} \mathrm{C}$ and $46^{\circ} \mathrm{C}$ is known as 'Mild Hyperthermia'. 9 In this temperature range, the treatment can selectively target and damage the cancerous tissue, leaving normal tissue intact (Figure 1). Contemporary therapeutic modalities for localised mild hyperthermia involve the use of a colloid of magnetic nanoparticles to induce the temperature increase, hence the term 'Magnetic Fluid Hyperthermia' (MFH). ${ }^{10}$ The magnetic properties of magnetic susceptibility, magnetic hysteresis and the Curie temperature $\left(T_{c}\right)$ are of interest in this work as they help to characterise the magnetocaloric effect (MCE) responsible for the temperature change. ${ }^{11-13}$ The MCE arises when certain magnetic materials are placed in a magnetic field and there is an associated change in temperature. ${ }^{14}$

In the presence of an alternating magnetic field, three forms of energy dissipation may contribute to the MCE where an

\footnotetext{
a. School of Chemistry and Chemical Engineering, Queen's University Belfast, Stranmillis Road, Belfast BT9 5AG, UK.

b. School of Mathematics and Physics, Queen's University Belfast, University Road, Belfast BT7 $1 N N, U K$.

Electronic Supplementary Information (ESI) available: [details of any supplementary information available should be included here]. See DOI: 10.1039/x0xx00000x
}

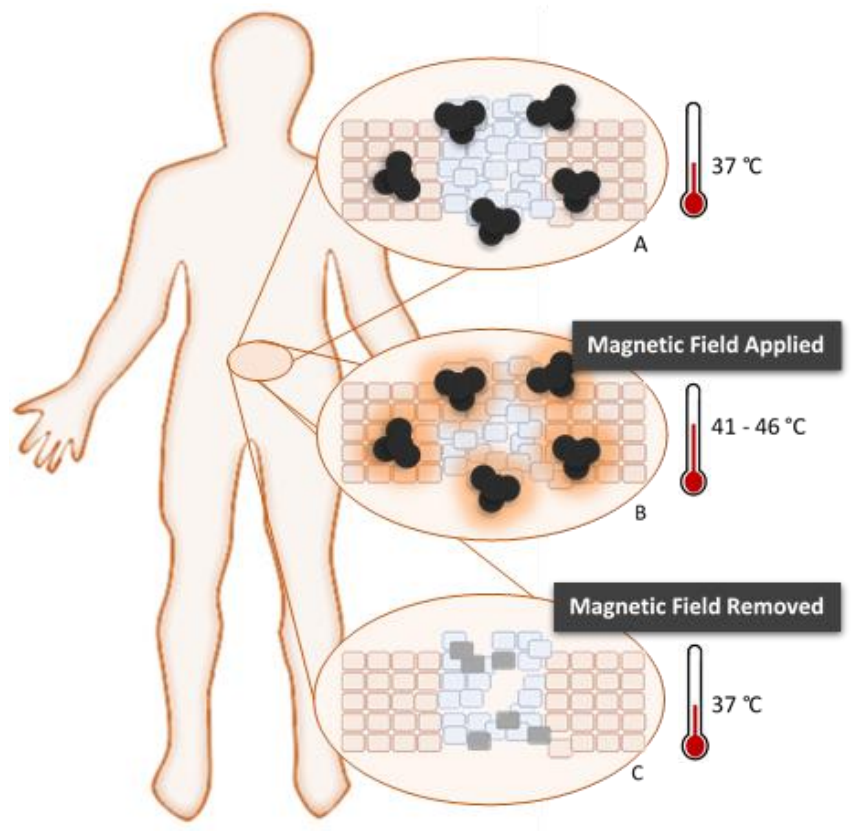

Figure 1 - Scheme depicting Magnetic Fluid (Mild) Hyperthermia treatment for cancer showing: (A) regularly shaped normal tissue (orange) and irregular cancerous tissue (blue) surrounded by clustered magnetic nanoparticles. (B) effect ossociated temperature rise and (C) effect of the applied alternating magnetic field on the survival of the different tissues. The applied field in and associated temperature rise has caused damage to some of the cancerous cells (shown in grey), but no damage to the normal tissue.

increase in temperature is observed: Brownian rotations, Neél rotations and magnetic hysteresis (shown in S.I. Figure S1). ${ }^{11}$ 
Materials which do not require high fields and low temperature to reach their saturation magnetisation $\left(M_{s}\right)$ are desirable for MCE biomedical application such as MFH. ${ }^{10} \mathrm{~A}$ high $\mathrm{M}_{\mathrm{s}}$ will mean that the material is more responsive to the applied field, and will promptly switch the direction of magnetisation (with respect to its crystal lattice) with an alternating magnetic field, resulting in greater heating losses via Brownian rotations, Neél rotations and in some cases, magnetic hysteresis.

The use of magnetic nanoparticles (MNPs) in magnetic fluid hyperthermia localises the heat to the cellular level. ${ }^{15}$ The three main requirements of the nanoparticles used to mediate hyperthermia treatment are: they should be biocompatible, have a Curie temperature $\left(T_{c}\right)$ within the therapeutic range of mild hyperthermia $\left(41{ }^{\circ} \mathrm{C}\right.$ to $46^{\circ} \mathrm{C}$ ) and have a high Specific Absorption Rate (SAR). ${ }^{16}$ The nanoparticles must be also be uniform in shape and monodisperse so as to ensure homogeneity and thus more consistent heating effects. ${ }^{17}$ Nanoparticles in the region of $100 \mathrm{~nm}^{18,19}$ are desirable for the purposes of MFH as they may be able to escape through the leaky tumour vasculature in order to localise the hyperthermic effects to the cellular level, improving the efficacy of the treatment. ${ }^{18,19}$ The development and characterisation of LSMO materials with $T_{c}^{\prime}$ 's within the therapeutic range of mild hyperthermia and high SARs is dealt with in this work.

The versatility of LSMO lies with the ability of its perovskite structure to accommodate different cations into the crystal lattice (shown in S.I. Figure S2). ${ }^{20}$ The parent compound, lanthanum manganate (LMO), is typically rhombohedral in crystal structure with space group $\mathrm{R}-3 \mathrm{C}$ and is non-magnetic at room temperature. ${ }^{21}$ Lanthanum ions occupy the A-site, with smaller manganate ions occupying the central B-site and oxide ions on the faces. Substituting lanthanum A-site ions for strontium in small amounts causes distortions of the crystal structure. The distortion of the crystal structure is both as a result of the increasing radii of the A-site and also the change in valency of the $B$-site manganese ion induced by the replacement of $\mathrm{La}^{3+}$ with $\mathrm{Sr}^{2+}$ at the A-site. The change in crystal structure leads to an incredibly rich phase diagram for LSMO shown in Figure 2, ranging from antiferromagnetic insulators to ferromagnetic insulators and ferromagnetic metals, which is why the material has been investigated for the wide variety of aforementioned applications. ${ }^{22}$ The phase descriptors refer to both the magnetic (anti-ferromagnetic, ferromagnetic or charge ordered), and electric properties (metallic or insulator) of the material. This work deals with LSMO in the ferromagnetic metallic region at a range of dopant levels $(x=0.25,0.35$ or 0.4$)$. Magnetic properties arise in manganates through a form of magnetic exchange known as the double-exchange (DE) mechanism shown in S.I. Figure S3, where an electron is resonantly transferred from $\mathrm{Mn}^{3+}$ to $\mathrm{O}^{2-}$, whilst one is transferred from $\mathrm{O}^{2-}$ to $\mathrm{Mn}^{4+} .{ }^{23}$ The extent of magnetic exchange via this mechanism is thought to vary based on the extent of orbital overlap, with bond lengths and angles being critical parameters. ${ }^{24,25}$ A preliminary consideration of the manganese valencies in S.I. Figure S3 would infer that increasing the proportion of $\mathrm{Mn}^{4+}: \mathrm{Mn}^{3+}$ towards a $1: 1$ ratio would favour the double exchange mechanism. However manganate crystal

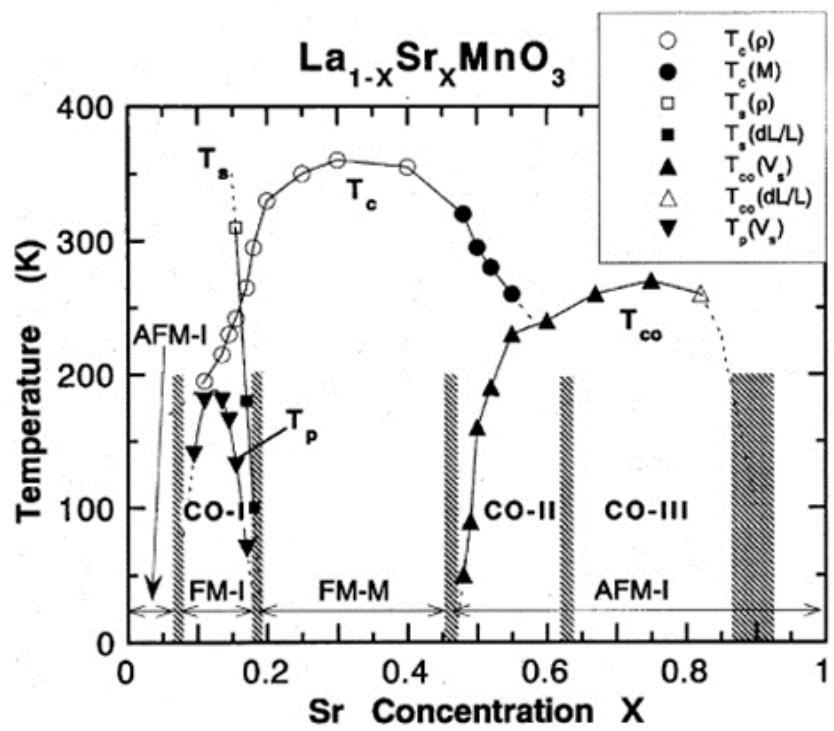

Figure 2 - Phase diagram for lanthanum strontium manganate reproduced from work by Majumdar et. al. 22 Phases include charge-ordered (CO), antiferromagne (AF), ferromagnetic metal (FM), and ferromagnetic insulator (FI). The unlabelled region of the phase diagram has neither magnetic nor charge order. The suffixes refer to the electrical conductivity of the material using the descriptions of metal (M) and insulator (I).

structures are also associated with Jahn-Teller distortions, which involve either contraction or elongation of bond lengths and angles, and are less prevalent with increasing strontium dopant levels in LSMO. $\mathrm{Mn}^{3+}$ in LMO is Jahn-Teller active due to its four valence electrons in a high spin state occupying nondegenerate orbitals. ${ }^{26}$ As the level of $\mathrm{Sr}^{2+}$ dopant increases in LSMO, the level of $\mathrm{Mn}^{4+}$ also increases. $\mathrm{Mn}^{4+}$ is a non JahnTeller active ion because its three valence electrons occupy degenerate $t_{2 g}$ orbitals. Jahn-Teller distortion occurs where initial degeneracy in either the $t_{2 g}$ or $e_{g}$ orbitals is removed by the compounds undergoing a geometrical transformation to lower the energy of the structure. ${ }^{27}$ This geometrical distortion manifests itself as either a structural compression or elongation. As the proportion of $\mathrm{Sr}^{2+}$ and $\mathrm{Mn}^{4+}$ increases, the proportion of $\mathrm{La}^{3+}$ and $\mathrm{Mn}^{3+}$ decreases meaning that Jahn-Teller distortion will be less effective at increasing the $\mathrm{Mn}^{3+}-\mathrm{O}^{2-}-\mathrm{Mn}^{4+}$ bond angle towards $180^{\circ}$ for optimal exchange. For this reason, the optimal ferromagnetic properties do not occur with a $1: 1$ ratio for $\mathrm{Mn}^{3+}: \mathrm{Mn}^{4+}{ }^{27}$

As can be seen in Figure 2, the optimal ferromagnetic metallic properties of LSMO are likely to occur at approximately a dopant level of $x=0.3$, as this is where the highest ferromagnetic to paramagnetic transition temperature or $T_{c}$ is observed. ${ }^{22}$ The greater the $T_{c}$, the more thermal energy is required to disrupt the magnetic ordering (i.e. the $D E$ mechanism) inducing the ferromagnetic to paramagnetic transition, and hence the greater the extent of magnetic ordering present. Thus the magnetic and electronic properties of LSMO are extremely sensitive to structural changes, leading to the variety of phases seen in Figure 2 (i.e. charge-ordered, anti-ferromagnetic insulator, ferromagnetic metal and ferromagnetic insulator). In altering the crystal structure of the material, it is possible to obtain a material with specific desired physical properties such as MCE, magnetic susceptibility and $T_{C}$, 
Table 1 - Variations in crystallite size and particle morphology of LSMO with varying methods from literature. Crystallite diameters presented are based on the unrefined diffraction data.

$\begin{array}{ccc}\begin{array}{c}\text { Ref } \\ \text { No. }\end{array} & \text { Authors } & \text { Type of Synthesis } \\ 36 & \text { Ahmad, M.A. et al. } & \text { Gel Combustion } \\ 37 & \text { da Conceição, L. et al. } & \text { Sol-gel } \\ 34 & \text { da Conceição, L. et al. } & \text { Solid State } \\ 38 & \text { da Conceição, L. et al. } & \text { Gel Combustion } \\ 39 & \text { Das, S. et al. } & \text { Pyrophoric } \\ 40 & \text { Gaudon, M. et al. } & \text { Sol-gel } \\ 41 & \text { Guo, R.S. et al. } & \text { Sol-gel } \\ 42 & \text { Huang, Y. et al. } & \text { Sol-gel } \\ 43 & \text { Kuznetsov, A.A. et al. } & \text { Freeze-drying } \\ 7 & \text { Kaman, O. et al. } & \text { Sol-gel } \\ 44 & \text { Lipham, N.D. et al. } & \text { Sol-gel } \\ 32 & \text { McBride, K. et al. } & \text { Sol-gel } \\ 45 & \text { Moreira, M.L. et al. } & \text { Sol-gel } \\ 8 & \text { Pollert, E. et al. } & \text { Sol-gel } \\ 46 & \text { Thorat, N.D. et al. } & \text { Gel Combustion } \\ 47 & \text { Zhang, Q. et al. } & \text { Solid State }\end{array}$

whilst maintaining its chemical and physical stability. ${ }^{28,29}$ Even slight differences in the proportion of strontium dopant in LSMO changes the bond lengths and angles, and significantly alters the Curie temperature $\left(T_{c}\right)$. For example, $\mathrm{La}_{0.9} \mathrm{Sr}_{0.1} \mathrm{MnO}_{3}$ has a $\mathrm{T}_{\mathrm{c}}$ of $-13{ }^{\circ} \mathrm{C}$ which increases to $77^{\circ} \mathrm{C}$ for $\mathrm{La}_{0.8} \mathrm{Sr}_{0.2} \mathrm{MnO}_{3}{ }^{6}$ Previous studies which have illustrated the tunable structureproperty relationship of LSMO perovskites have mainly investigated the effect of altering composition ${ }^{6,30-32}$ or particle size $^{33}$ on the magnetic properties of the LSMO crystallite clusters. It has also been shown that the actual method of synthesis employed will affect the physical properties such as the electrical conductivity of the material. ${ }^{34}$ Table 1 shows that there appears to be considerable variation in the size and morphology of LSMO particles obtained using various synthetic methods, which may in part lead to observed differences in physical properties such as the aforementioned electrical conductivities. As particle size and morphology are shown in Table 1 to be parameters which in part determine the physical properties of a material, it also follows that the degree of crystallinity is also an important parameter. The link between the degree of crystallinity with variation in the extent of magnetic contribution is put forward in a model by Zhang et al. ${ }^{35}$ In this model, it is proposed that magnetic nanoparticles were composed of a ferromagnetic core surrounded by an antiferromagnetic shell. It was also thought that a change in crystallinity would alter this ferromagnetic to antiferromagnetic ratio, thereby leading to a change in the observed MCE which is further investigated in this work vide priori.

In addition to the variation of crystallite sizes and size distributions of LSMO materials shown in Table 1 , there is also considerable variation in the much larger agglomerate crystal size. (The values are not directly comparable due to some samples undergoing milling and so are not presented.) The desired agglomerate size for $\mathrm{MFH}$ is of the order of $100 \mathrm{~nm}$ to

$\begin{array}{ccc}\text { Using } & \text { Particle } & \begin{array}{c}\text { Crystallite Size } \\ (\mathrm{nm})\end{array} \\ \text { Citrate-Nitrate } & \text { Shape } & 22 \\ \text { Ethylene glycol } & \text { Spherical } & 19-28 \\ \mathrm{~N} / \mathrm{a} & \text { Spherical } & 12-20 \\ \text { Citrate-Nitrate } & \text { Spherical } & 15-20 \\ \text { Nitrates and PVA/TEA } & \text { Various } & 10-13 \\ \text { Citric acid } & \text { Spherical } & 50-110 \\ \text { Glycine-Nitrate } & \text { Various } & 50-60 \\ \text { EDTA and ammonia } & \text { Cubic } & - \\ \text { N/a } & \text { Spherical } & - \\ \text { Citric acid and } \mathrm{H}_{2} \mathrm{O}_{2} & \text { Various } & 20 \\ \text { Citric acid } & \text { Spherical } & 11-17 \\ \mathrm{H}_{2} \mathrm{O}_{2} \text { and ammonia } & \text { Spherical } & 62-165 \\ \text { Citric acid } & \text { Various } & 20-95 \\ \text { Citric acid } & \text { Spherical } & 30-49 \\ \text { Citrate-Nitrate } & \text { Spherical } & 40 \\ \text { Mechanochemical } & \text { Spherical } & -\end{array}$

allow for the intra-venous delivery of the magnetic nanoparticles. ${ }^{18,19}$ The agglomerates obtained by our group using the conventionally heated peroxide sol-gel synthesis were two orders of magnitude greater. ${ }^{32}$ Control of the agglomerate crystal size is essential for the viable use of LSMO in potential biomedical applications such as MFH. ${ }^{10}$ However, it is imperative that the physical properties (such as magnetic susceptibility) are not impaired when size reduction strategies such as milling or silica encapsulation are employed as this would reduce the efficacy of the material as a mediator for $\mathrm{MFH} .{ }^{19}$ One strategy which may help satisfy these requirements is to employ the assistance of Microwave (MW) irradiation during the synthesis. A few methods demonstrate the advantageous use of MWs in the synthesis of LSMO, including a MW-hydrothermal method ${ }^{36}$, MW sintering ${ }^{37}$, and the use of combined MW Pechini synthesis and calcination. ${ }^{38}$

Microwave (MW) irradiation is a promising materials processing method, offering advantages in terms of shorter reaction times, smaller particle sizes and narrower size distributions compared with conventional methods of heating. ${ }^{20}$ Electrical dipole moments found in polar materials (e.g. water or metal oxides) will align or couple with an electromagnetic field in the $\mathrm{MW}$ region. The alternating $\mathrm{MW}$ radiation causes molecules to rotate with the electromagnetic field, which causes friction and collisions of the molecules and their surroundings. ${ }^{39,40}$ There are two mechanisms which contribute to the net MW heating: 1) solvent heating (water); and 2) particle heating (perovskite precursors). In the peroxide sol-gel synthesis used in this work, the dominant heating mechanism is dielectric solvent heating due to the hydrogen peroxide and water mixture having much greater dielectric constants $(70-80)^{41}$ than the perovskite precursors (lanthanum oxide $(20-50)^{42}$, strontium carbonate $(9)^{43}$ and manganese carbonate $\left.(1-5)^{44}\right)$. As the energy dissipation arises from within the core of the reactant mixture, a more regular temperature 
distribution can be achieved ${ }^{45-47}$ compared with conventional heating ${ }^{48-50}$ without damage due to thermal shock. ${ }^{40} \mathrm{MW}$ syntheses have been proven to be efficient and selective, and as a result they are being investigated by many researchers in order to improve a wide range of reactions. ${ }^{49}$

The use of MW irradiation as an alternative heating method in established syntheses is proving to be advantageous. MWassisted solid-state syntheses or calcinations have proven to be a popular application of the technology, with temperatures in excess of $1000{ }^{\circ} \mathrm{C}$ reported to be achieved in a matter of a few minutes producing the desired crystalline product such as in the decrystallisation of oxides which occurs in seconds. ${ }^{51} \mathrm{MW}$ syntheses may be used to synthesise metal oxides such as perovskites in solid-state reactions due to their inherent large dielectric constant and high dielectric loss. ${ }^{52}$ This means they behave as a passive heating element when a $\mathrm{MW}$ field is applied. MW-assisted syntheses have been shown to be useful in producing materials which have a purer monocrystalline phase, in addition to allowing reasonable control over the crystallite growth and crystal structure depending on the reaction conditions. In the synthesis of the $\mathrm{LaFeO}_{3}$ for example, a smaller and more crystalline product was obtained following four minutes of MW irradiation compared with conventional methods. ${ }^{53}$ Improved crystallinity was also achieved with postcalcination $\mathrm{MW}$ treatment in the synthesis of $\mathrm{CaCu}_{3} \mathrm{Ti}_{4} \mathrm{O}_{12} \cdot{ }^{54}$ Greater selectivity of crystal structure was also observed in the $\mathrm{MW}$-hydrothermal synthesis of $\mathrm{BaTiO}_{3}$ where a greater proportion of the tetragonal crystal phase was obtained using the MW synthesis than was observed with conventional heating methods. ${ }^{55}$

So far, the focus of MW investigations have been on the increased rates of reaction in addition to the degree of crystallinity in the materials. To the knowledge of the authors, there is little information regarding the impact of MW irradiation on the magnetic properties of highly crystalline materials synthesised using this technology. However, Yu et. al recognised that there were enhanced dielectric properties of the perovskite $\mathrm{CaCu}_{3} \mathrm{Ti}_{4} \mathrm{O}_{12}$ when synthesised using $\mathrm{MW}$ heating. ${ }^{56}$ As there is a great deal of similarity in how dielectric and magnetic properties arise in materials ${ }^{39,40}$ we could also expect enhanced magnetic properties (magnetic susceptibility, magnetic hysteresis and magnetocaloric effect) of a similar material processed using $\mathrm{MW}$ technology. With this in mind, this investigation aims to look at the effect of the use of $\mathrm{MW}$ irradiation in a modified peroxide sol-gel synthesis on the magnetic properties of lanthanum strontium manganate (LSMO) compared with conventional heating. To the best knowledge of the authors, this is the first time that $\mathrm{MW}$ irradiation has been applied to the peroxide sol-gel synthesis. Initially this work is concerned with improving the structural and magnetic properties of LSMO at a range of dopants $(x=0.25$, 0.35 and 0.4 ) using the $\mathrm{MW}$-assisted peroxide sol-gel synthesis in order to confirm the most suitable stoichiometry of LSMO for use in MFH (Section 3.1). The conditions for the MW-assisted peroxide sol-gel synthesis of this material are then investigated in order to optimise the MCE (Section 3.2). The assistance of MW irradiation during the material synthesis is expected to increase the ferromagnetic to anti-ferromagnetic ratio of LSMO particles by improving the crystallinity of the material as previously mentioned. ${ }^{35}$ In doing so, not only is the hope to increase the ferromagnetic composition of the material in order to improve its efficacy as a mediator for MFH, but the increase in crystallinity would also hopefully lead to more discrete aggregates which could be more effectively broken down to a suitable size (order of $100 \mathrm{~nm}$ ) for MFH by grinding.

\section{Experimental}

\subsection{Material synthesis}

Powder precursors (obtained from Sigma Aldrich); lanthanum (III) oxide, $\geq 99.9 \%\left(\mathrm{La}_{2} \mathrm{O}_{3}\right)$; manganese(II) carbonate, $\geq 99.9 \%$ trace metals basis; and strontium carbonate, $\geq 99.9 \%$ trace metals basis; were mixed in appropriate proportions in order to obtain the desired stoichiometric ratios. No thermal pretreatments including drying were used prior to weighing, as the lanthanum oxide was stored in a cold dry place in accordance with storage instructions. XRD analysis of the powder showed it to have remained free from lanthanum hydroxide impurities which would have necessitated thermal pre-treatment. The method for the conventionally heated peroxide sol-gel synthesis is reported elsewhere. ${ }^{32}$ For the MW samples (LSMO25MW, LSMO35MW and LSMO4OMW); while stirring, $1.25 \mathrm{~mL}$ of hydrogen peroxide (30 vol\% - Fisher Scientific) was added slowly, followed by addition of 3 drops $(\sim 120 \mu \mathrm{l})$ of ammonium hydroxide $\mathrm{NH}_{4} \mathrm{OH}$ (25 vol\%, Sigma Aldrich). Following the cessation of bubbling, the mixture was transferred to a closed, but not sealed, $10 \mathrm{~mL}$ microwave tube and placed in the microwave holder. After stirring at $70^{\circ} \mathrm{C}$ for 2 hours in the microwave (CEM Discover $2.45 \mathrm{GHz}$ ) set to $100 \mathrm{~W}$, the resulting solution was dried at $110{ }^{\circ} \mathrm{C}$ until dried gel formation, which occurred in 15 - 30 minutes. This process was repeated 30 times for each sample (LSMO25MW, LSMO35MW and LSMO4OMW), and the powder samples combined in order to obtain enough final product to conduct a Rietveld analysis on the powder diffraction data. The MW parameters were further investigated in order to optimise the magnetic heating properties of the material with the optimal dopant level (LSMO35MW). When the heating time was investigated in the $\mathrm{MW}$ synthesis of LSMO35MW, the precursors were heated at $100 \mathrm{~W}$ power for time intervals of 10 minutes, 30 minutes or 2 hours. When power was investigated in the MW synthesis of LSMO35MW, all precursors were heated for 2 hours at power settings of $50 \mathrm{~W}, 100 \mathrm{~W}$ or $200 \mathrm{~W}$. Where MW parameters (time and power) are optimised, the process is only repeated three times in order to produce enough material for repeat magnetic measurements and magnetic heating measurements. Subsequent calcination at $1100{ }^{\circ} \mathrm{C}$ for 16 hours was used to remove the residual carbon and obtain a crystalline product. All samples (presented in the Supplementary Information Table S1) were manually ground in an agate mortar and pestle before characterisation and testing.

The modified peroxide sol-gel synthesis was used in previous work ${ }^{32}$ to produce LSMO materials at a variety of dopant levels using conventional heating i.e. a hot-plate and 
stirrer. These conventionally synthesised materials are used as a point of comparison to show the improvement in the structural and magnetic properties of LSMO when MW heating is used in this work. The modified peroxide sol-gel synthesis ${ }^{32}$ has not been carried out using $\mathrm{MW}$ irradiation before, and adjustments to the reactant mixture previously used were necessary to make it suitable for use in the industrial $\mathrm{MW}$. It has been conducted by adding alkaline hydrogen peroxide directly to the metal oxide and metal carbonate precursors, rather than to an aqueous suspension of the precursors. The absence of additional water in the MW-assisted synthesis is to prevent the superheating of water and reduce the total volume in the microwave tube in order to prevent damage to the IR probe which controls the temperature programme. In order to compare the LSMO samples which have been conventionally heated (i.e. heated with a hot plate) with those irradiated with MWs, a sample (LSMO35 - RedVol) which has been conventionally heated but without additional water was also synthesised. Supplementary Information Table S2 shows all the produced samples and sample names to be used for the remainder of the report. All of the materials with the -Con suffix were synthesised using conventional heating ${ }^{32}$, and -RedVol indicates the omission of the water from the conventional synthesis. This allows us to separate the contribution of reducing the reactant volume from the contribution of $\mathrm{MW}$ irradiation, to the physical properties (e.g. MCE). Initially, LSMO samples were synthesised at dopant values $x=0.25,0.35$ and 0.4 to confirm that LSMO35 was in fact the target material which should be optimised. Then the process was optimised by synthesising LSMO35 at various time and power settings. A structural characterisation of the crystalline phases for the LSMO samples (LSMO25, LSMO35 and LSMO40) obtained using the MW assisted synthesis is investigated, along with a study of the physical properties of interest to a mediator for MFH with the optimal dopant of LSMO35, i.e. the magnetic susceptibility, magnetic hysteresis and magnetocaloric effect.

The MW irradiation of the sample is controlled by an external temperature control which limits the MW power depending on the measured external temperature of the tube. As a result, the MW irradiation of the sample is not constant; initial irradiation of a proportion of the set MW power will be followed by a periodic pulse of a much smaller power setting to maintain the set temperature. The upper temperature limit for this style of $\mathrm{MW}$ is $300{ }^{\circ} \mathrm{C}$ so it is suitable for the main material synthesis but not the final calcination step.

\subsection{Characterisation}

The crystal structures making up each powder sample were determined using XRD; Panalytical X'Pert was used to collect the $X$-ray diffraction patterns between 20 and 80 degrees and $X^{\prime}$ Pert High Score Plus was used to conduct a Rietveld analysis for LSMO25MW, LSMO35MW and LSMO4OMW samples. The Rietveld refinement approach is presented in a prior work. ${ }^{32}$ The presented crystallite sizes may also vary depending on the method of calculation. In cases where the crystallite diameter is calculated for a material which is both polycrystalline and polymorphic, the Full-Width at Half Maximum (FWHM) value for the most intense reflection which is typically used in the Scherrer equation, is a convolution of the reflections of the constituent phases and can therefore lead to an unrepresentative crystallite diameter. For example, in the calculation of crystallite diameter from the un-refined diffraction pattern of $\mathrm{La}_{0.6} \mathrm{Sr}_{0.4} \mathrm{MnO}_{3}$ the average crystallite size is $61.9 \mathrm{~nm}^{32}$, whereas calculating this following Rietveld refinement gives orthorhombic and rhombohedral crystallite diameters of 16.5 and $37.4 \mathrm{~nm}$ respectively. ${ }^{57}$ The crystallite diameters have been calculated for the data post- Rietveld refinement and are shown in Table 2, however the average crystallite size was calculated on the data pre- Rietveld refinement in order to calculate the magnetically dead layer in Section 3.2.

Rietveld refinement of powder diffraction pattern for LSMO25MW, LSMO35MW and LSMO40MW allowed electron density maps to be generated. The electron density maps in Section 3.1 are Fourier difference maps, which identify differences between the observed and calculated model crystal structures. The maps are used to optimise the model structure parameters in an iterative background process, but are displayed for the reader in this work in order to critically evaluate the reliability of the individual crystal structures and better elucidate any trends in the calculated $\mathrm{Mn}-\mathrm{O}$ bond lengths or $\mathrm{Mn}-\mathrm{O}-\mathrm{Mn}$ bond angles (generated from the refined diffraction data). 'Visualization for Electronic and Structural Analysis' (VESTA) software was also used to visualise the isolated crystal phases following Rietveld analysis. ${ }^{58}$ The morphology of the resulting samples was studied using SEM (eSEM - FEI Quanta FEG- Environmental SEM Oxford Ex-ACT). The stoichiometry of the synthesised materials was then confirmed using eSEM-EDX with the Aztec based XACT system. Atomic percentage was determined using the relative ratio of La to Sr elements in each sample, with the uncertainty for the data values at the $\pm 1 \%$ level using a cobalt standard.

For all samples, magnetic heating experiments were conducted on $10 \mathrm{mg} \mathrm{mL}^{-1}$ aqueous suspensions of the MNPs in non-adiabatic conditions using an AC generator (Easyheat 0112) in conjunction with a water-cooled induction coil at a frequency of $175 \mathrm{kHz}$ and field amplitude of $10.95 \mathrm{kAm}^{-1}$. The set-up is shown elsewhere. ${ }^{32}$ An IR temperature probe (Optocon AG) FOTEMP 1 coupled to a (Optocon AG) TS2/2 sensor was used to record the magnetic heating measurements from which the SAR was calculated using the corrected slope method (S.I. Equation 1). ${ }^{59}$ A superconducting quantum interference device (SQUID) magnetometer (Quantum Design MPMS XL) was used to measure the magnetic susceptibility and magnetisation hysteresis for the LSMO35 samples synthesised at various power settings. Field dependent magnetisation measurements were conducted in the range $-10,000 \leq \mathrm{H} \leq 10,000 \mathrm{Oe}$, at $100 \mathrm{~K}$ and $200 \mathrm{~K}$. Susceptibility measurements were conducted in field cooled (FC) and zero field cooled (ZFC) conditions between 100 and $370 \mathrm{~K}$, at $500 \mathrm{Oe}$. It is also important to note that for the magnetomety measurements, LSMO35-MW unusually appeared to melt the gelatine capsules typically used for these measurements. As a result all the magnetic measurements 
Table 2 - Refined lattice parameters resulting from Rietveld analysis for LSMO materials synthesised used MW-assistance or using conventional heating from previous work ${ }^{32}$ denoted by '-MW' and '-Con' suffixes respectively. ( $R_{w}$ is the weighted R-value, $R_{\exp }$ is the expected R-value, $R_{\text {bragg }}$ is the specific $R$-value for a particular phase in the refinement, Gof is the Goodness of Fit). Angles $\alpha, \beta$ and $\gamma$ were were not allowed to vary during the refinement. The corresponding angles were $\alpha=\beta=\gamma=90^{\circ}$ for Pnma and $\alpha=\beta=90^{\circ}$ and $\gamma=120^{\circ}$ for R-3c. Crystallite size is estimated with the Scherrer equation, using the most intense reflection for each phase. Error values were calculated using the crystallite diameters calculated from three of the most intense reflections for each crystalline phase. The numbers in parentheses are the estimated uncertainties relative to the last significant digits of the numerical values.

\begin{tabular}{|c|c|c|c|c|c|c|c|c|c|c|c|}
\hline Sample & $\begin{array}{l}\text { Space } \\
\text { group }\end{array}$ & a / $\AA$ & b / $\AA$ & $c / \AA ̊$ & $V / \AA^{3}$ & $\mathrm{R}_{\mathrm{w}}$ & $R_{\text {exp }}$ & $R_{\text {bragg }}$ & Gof & $\begin{array}{c}\text { Wt. } \\
\text { percent. } \\
/ \%\end{array}$ & $\begin{array}{c}\text { Crystallite } \\
\text { diameter } \\
(\mathrm{nm})\end{array}$ \\
\hline \multirow{2}{*}{ LSMO25-Con } & Pnma & $5.4373(16)$ & $7.7984(21)$ & $5.4331(15)$ & $230.4(2)$ & \multirow{2}{*}{10.7} & \multirow{2}{*}{3.5} & 4.2 & \multirow{2}{*}{9.3} & $8.2(8)$ & $164.5(145)$ \\
\hline & $R-3 c$ & $5.5146(5)$ & $5.5146(5)$ & $13.3552(13)$ & $351.7(1)$ & & & 2.6 & & $91.8(6)$ & $41.4(0)$ \\
\hline \multirow{2}{*}{ LSMO35-Con } & Pnma & $5.4482(33)$ & $7.7433(31)$ & $5.4678(39)$ & $230.4(4)$ & \multirow{2}{*}{8.2} & \multirow{2}{*}{3.8} & 18.8 & \multirow{2}{*}{4.6} & $19.7(8)$ & $24.3(0)$ \\
\hline & $\mathrm{R}-3 \mathrm{c}$ & $5.5120(3)$ & $5.5119(3)$ & $13.3546(8)$ & $351.4(1)$ & & & 28.8 & & $80.3(9)$ & 48.4(1) \\
\hline \multirow[b]{2}{*}{ LSMO40-Con } & Pnma & $5.4482(30)$ & $7.7501(43)$ & $5.4562(36)$ & $230.4(4)$ & \multirow[b]{2}{*}{6.1} & \multirow[b]{2}{*}{2.7} & 29.5 & \multirow{2}{*}{5.0} & $31.2(13)$ & $20.4(0)$ \\
\hline & $R-3 c$ & $5.5109(4)$ & $5.5109(4)$ & $13.3543(13)$ & $351.2(1)$ & & & 9.6 & & $68.8(13)$ & $46.3(1)$ \\
\hline \multirow{2}{*}{ LSMO25-MW } & Pnma & $5.1694(2)$ & $8.3922(2)$ & $5.7181(7)$ & $248.1(0)$ & \multirow{2}{*}{5.8} & \multirow{2}{*}{4.3} & 40.5 & \multirow{2}{*}{1.8} & $12.1(13)$ & $106.5(2)$ \\
\hline & $R-3 c$ & $5.5054(3)$ & $5.5054(3)$ & $13.3571(7)$ & $350.6(1)$ & & & 6.6 & & $87.9(8)$ & $51.2(0)$ \\
\hline \multirow{2}{*}{ LSMO35-MW } & Pnma & $5.0948(248)$ & $9.1774(380)$ & $6.9987(230)$ & $327.3(11)$ & \multirow{2}{*}{7.8} & \multirow{2}{*}{5.8} & 31.2 & \multirow{2}{*}{1.8} & 19.0(9) & $8.8(7)$ \\
\hline & $R-3 c$ & $5.4940(4)$ & $5.4940(4)$ & $13.3477(12)$ & $348.9(1)$ & & & 7.57 & & $81.0(0)$ & $54.6(1)$ \\
\hline \multirow{2}{*}{ LSMO40-MW } & Pnma & $5.4635(11)$ & $7.6864(12)$ & $5.5185(10)$ & $231.7(1)$ & \multirow{2}{*}{5.9} & \multirow{2}{*}{4.0} & 24.9 & \multirow{2}{*}{2.2} & $14.7(7)$ & $66.2(3)$ \\
\hline & $R-3 c$ & $5.4919(4)$ & $5.4919(4)$ & $13.3474(16)$ & $348.6(1)$ & & & 8.2 & & $85.3(8)$ & $33.8(1)$ \\
\hline
\end{tabular}

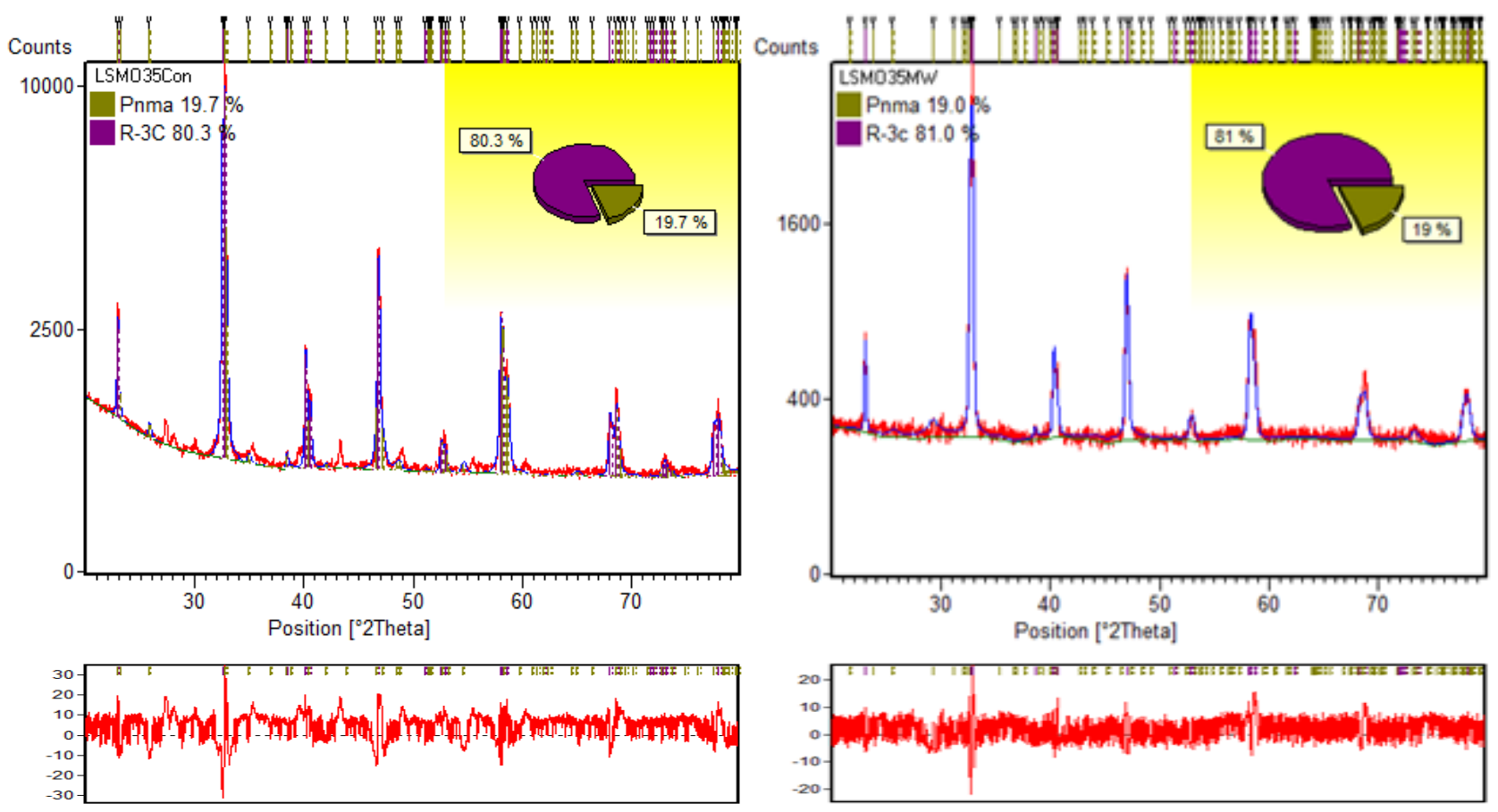

Figure 3 - Rietveld analyses of X-ray Diffraction Patterns of a selection of LSMO35 materials (a) LSMO35-Con (reproduced from previous work by this group ${ }^{32}$ ) and (b) LSMO35-100W to quantify the relative weight percentages of orthorhombic (Pnma) and rhombohedral (R-3c) crystalline phases. The red line below each diffraction pattern is the difference plot which gives an indication of the variation between the calculated and experimental data. *synthesised for the same length of time ( 2 hours) as the conventional synthesis and at $100 \mathrm{~W}$.

presented were measured in Peek capsules to prevent any damage to the SQUID. Results obtained using LSMO35-Con in gelatine capsules were reproducible when the Peek capsules were used and so comparison can be reliably made between the conventional synthesis used in previous work ${ }^{32}$ and the MWirradiated synthesis used here.

\section{Results and discussion}

3.1 Improving Structural and Magnetic Properties of LSMO (x $=0.25,0.35$ and 0.4 ) using $\mathrm{MW}$ irradiation

\subsubsection{Structural Characterisation}

The MW-assisted peroxide sol-gel method successfully synthesised polycrystalline LSMO at a variety of dopant levels ( $x$ $=0.25,0.35$ and 0.4 ) as confirmed through the powder diffraction patterns in S.I. Figure S4 with reflections in keeping 
(a)

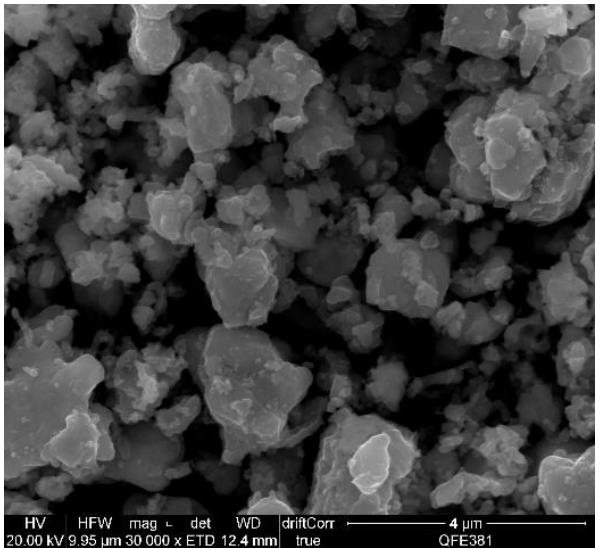

(c)

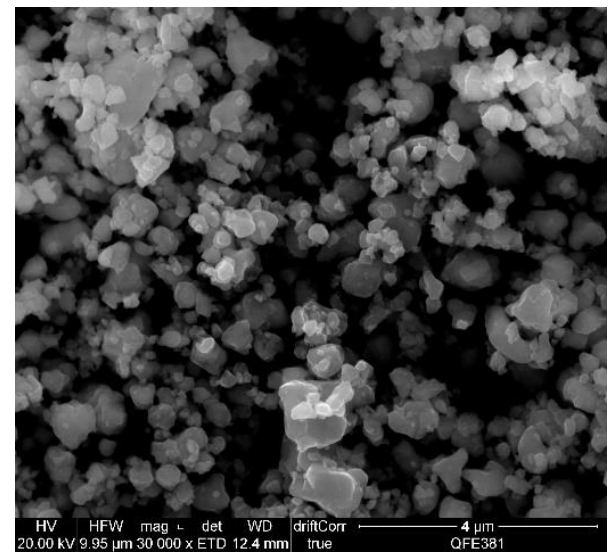

(b)

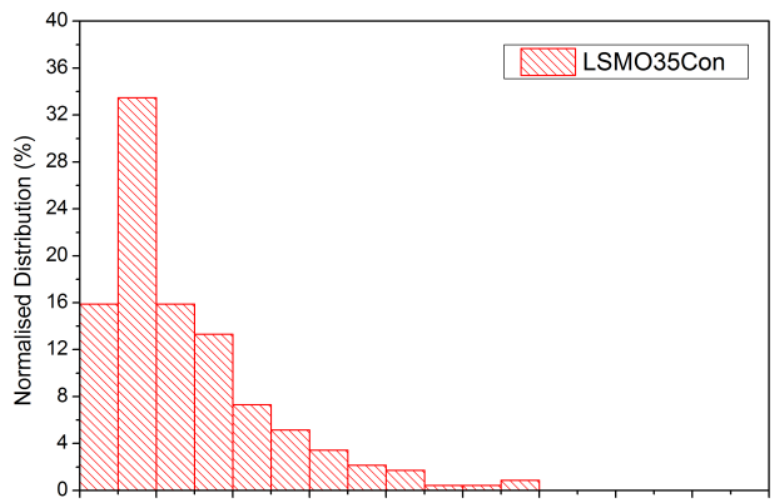

(d)

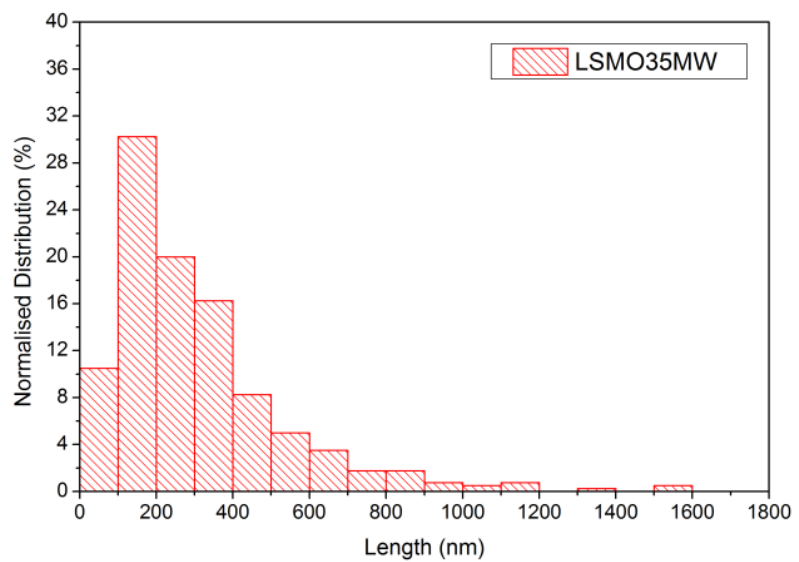

Figure 4 - Scanning Electron Micrographs of the materials (a) LSMO35Con (c) LSMO35MW synthesised at $100 \mathrm{~W}$ for 2 hours taken at 30,000 times magnification with (b) and (d) showing the respective normalised distributions of particle lengths for each sample.

with existing characterisations in prior work. ${ }^{32}$ Stoichiometries of the samples were confirmed using EDX and shown in the S.I. (Figure S5 - S7). All three diffraction patterns of the samples show a high degree of crystallinity, with a low background. This crystallinity, which is also evident in the scanning electron micrographs (SEMs also in S.I. Figure S4) is much greater than for the LSMO samples synthesised using conventional heating. This is further illustrated in the comparison of LSMO35 in Figure 4. ${ }^{32}$ Quantifying the crystallinity by using the ratio for the observed intensity of reflection compared with the intensity of the background is not appropriate here because the change in crystallinity has manifested in a change in the crystalline habits which would lead to a different distribution of intensities. Any reduction in crystallite size between the syntheses leads to broadening which would also affect the intensity of reflection. 60

The micrographs in S.I. Figure S4 of the LSMO samples $(x=$ $0.25,0.35$ and 0.4 ) show variation in particle sizes between samples of different dopant levels, with LSMO35-MW showing the smallest particle size. When the micrographs of LSMO materials synthesised using the MW-assisted method (S.I. Figure S4) are compared with samples from the conventional method of synthesis, ${ }^{32}$ not only do the MW samples show a higher degree of crystallinity, but the nanoparticles also appear to have greater uniformity in both in size and shape. This is promising as it means that size reduction strategies such as milling may be more effective to produce particles in the region of $100 \mathrm{~nm}^{18,19}$ suitable for the purposes of $\mathrm{MFH}$, and may also lead to materials with superior magnetic heating properties. The increasing crystallinity of the material according to Zhang et al.'s model may also have led to an increased the ferromagnetic to anti-ferromagnetic ratio, meaning we may see enhanced magnetic properties (the magnetic susceptibility, magnetic hysteresis and $\mathrm{MCE}$ ) shown through magnetic and induction heating measurements vide infra. ${ }^{35}$

The Rietveld analysis of the diffraction patterns showed that the MW-assisted synthesis used in this work, not only produced the same LSMO materials as in previous work, but also the same crystalline orthorhombic (Pnma) and rhombohedral (R-3c) polymorphs, with $\mathrm{R}-3 \mathrm{c}$ being the dominant geometry. ${ }^{32}$ Other minor phases which may be present in trace amounts (<0.1\%) include $\mathrm{La}_{2} \mathrm{O}_{3}, \mathrm{MnCO}_{3}$ and $\mathrm{SrMnO}_{3}$.

For ease of comparison, Table 2 shows the comparison of LSMO materials synthesised using this $\mathrm{MW}$-assisted method compared to the conventional synthesis. It is clear that for the samples synthesised using the MW-assisted method, there is a contraction in the unit cells along the a- and c - axes but an elongation of the material along the $b$ - axis. Overall, this actually leads to an increase in unit-cell volume for the MW synthesised samples compared with the conventionally synthesised samples. A larger unit cell volume for orthorhombic 
Table 3 - Fourier difference maps of a range of lanthanum manganates, which have been generated using $F_{\text {(obs) }}-F_{\text {(calc), }}$ following Rietveld analysis using High Score Plus of X-ray diffraction data. Colour maps are restricted to show the comparison of samples between -13 and +10 e $A^{-3}$.

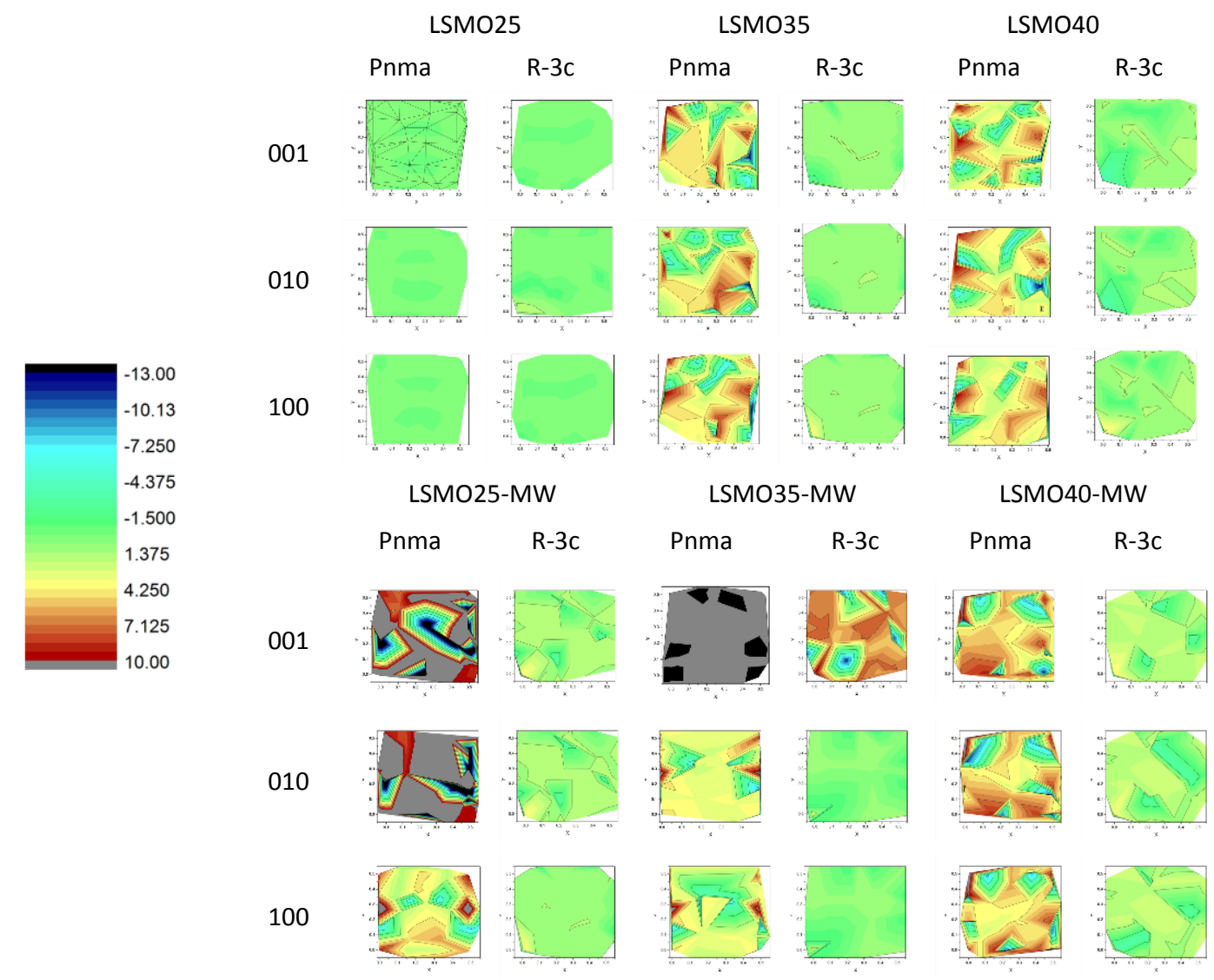

(Pnma) unit cells was shown to lead to smaller crystallite sizes in previous work. ${ }^{32}$ This is also true for LSMO25-MW and LSMO35-MW samples in this work when compared with their conventionally synthesised counterparts.

In previous work it was confirmed that the most suitable stoichiometry of LSMO synthesised using the modified peroxide sol-gel (conventional) synthesis for use in MFH was $\mathrm{La}_{0.65} \mathrm{Sr}_{0.35} \mathrm{MnO}_{3}{ }^{32}$. As a result, more detailed structural comparisons of LSMO35 synthesised using both the conventional (LSMO35-Con) and the MW-assisted peroxide solgel syntheses are shown (Figures 3 and 4 ) in order to better understand the structure-property relationship present in the samples, and if this differs depending on the synthetic method.

Figure 3 shows the X-ray diffraction patterns following Rietveld analyses for LSMO35 samples synthesised using (a) conventional heating and (b) MW heating synthesised for the same length of time ( 2 hours) as the conventional synthesis and at $100 \mathrm{~W}$. This $\mathrm{MW}$-assisted synthesised sample was chosen so as to represent the general improvement in structural and MCE properties (as seen in 3.2.2) which comes with using this method of synthesis, but also because this material rapidly heats in the linear regime to $43{ }^{\circ} \mathrm{C}$ meaning that it would be the most suitable for MFH without the danger of an extremely high maximum temperature as in the case of LSMO35-200W.
The summary of the refinements are presented in Table 2. The sample synthesised using MW irradiation shows a slight increase in orthorhombic crystal structure (Pnma) of $0.7 \%$ which we attribute to slight variations in the global stoichiometry of samples as shown in Table 2. The crystal structure of LSMO was previously shown to vary as a function of dopant by the authors. ${ }^{32}$ In that study, it was shown that as the level of strontium dopant increased, the weight percentage of the orthorhombic crystal structure was also shown to increase. When the LSMO35-MW sample is compared in terms of the exact stoichiometry (Table 2 and Supplementary Figure S10) against the range of doped LSMO materials synthesised using conventional heating in that work, the weight percentage of Pnma is in agreement with the polynomial line of best fit. Thus the slight difference in this quantification between LSMO35-MW and LSMO35Con (0.7\%) is therefore deemed to be a result of a slight variation in stoichiometry of the compound. This slight variation may be due to the batch nature of the MWassisted synthesis whereby multiple small scale reactions were combined in order to produce a sufficient quantity to conduct a Rietveld analysis. The difference in crystal structures as a result of a different type of syntheses in this instance is negligible; crystal structure is a function of dopant.

Due to the comparable orthorhombic and rhombohedral crystal structure distributions for the $\mathrm{MW}$ and conventionally 
synthesised samples, we would expect to see extremely similar physical properties such as the MCE investigated through the induction heating experiments and magnetic hysteresis. In spite of the similarities in crystal structure compositions (Pnma and $\mathrm{R}-3 \mathrm{c}$ geometries) there is one significant difference in the diffraction patterns. The straighter background with less noise in Figure 3(b) highlights that the degree of crystallinity in the MW sample is indeed greater; this is also confirmed by SEM.

The SEMs in Figure 4 show the morphology of LSMO35 samples for the synthesis routes employed: (a) Conventional peroxide sol-gel and (c) MW-assisted peroxide sol-gel. The LSMO samples first appear to be markedly different based on the synthesis route employed. The conventional synthesis in 4 (a) shows a highly polycrystalline sample with a wide range of agglomerate particle sizes and shapes (both spherical and platelike). The MW synthesis however in Figure 4 (c) appears to lead to a more crystalline material with smaller and more uniformly crystallised particles, both in terms of size and shape. However, on closer inspection shown by the histograms in Figures 4 (b) and (d), we see that the particle sizes have remained consistent between the samples with a mean particle length of approximately $300 \mathrm{~nm}$ in each case, but only a fraction have agglomerated in the LSMO35-MW sample (Figure 4 (c)) compared with the LSMO35Con sample (Figure 4 (a)). On further comparison of Figure 4 with Table 2, we see that the crystallite lengths from SEM are approximately five times greater than the calculated crystallite diameter from XRD.

Binner et al. postulated that rapid heating rates associated with $\mathrm{MW}$ heating may result in an increased rate of synthesis of titanium carbide due to an increased rate of diffusion of cations within the lattice. ${ }^{61}$ The increased rate of diffusion associated with MW heating may in part explain the improvement in crystallinity seen here in the LSMO35-MW sample. This increase in crystallinity may also cause a decrease in the size of the antiferromagnetic "magnetically dead layer" at the surface as nanoparticles become more discrete in keeping with Zhang's model. ${ }^{62} \mathrm{~A}$ decrease in the "magnetically dead layer" would lead to a greater ferromagnetic proportion of the material able to participate in the double-exchange mechanism. Thus leading to a greater value for saturation magnetisation, which in turn would mean that we could expect to see a greater MCE as observed through the induction heating experiments.

So far we have identified the crystal structure compositions and the degree of crystallinity for the various LSMO samples. We now aim to look in greater detail at the bond lengths and angles of the various crystal structures of LSMO at a variety of dopant levels $(x=0.25,0.35$, and 0.4$)$ in order to better relate their structures with their magnetic properties. Following Rietveld refinement of the powder diffraction patterns, it is possible to calculate the bond lengths and angles for the various LSMO materials. The quality of the Rietveld refinement must be taken into consideration when examining the dependent bond lengths and angles. The quality of a Rietveld refinement is typically described by a reliability marker known as the goodness of fit (Gof), similar to $\chi^{2}$; where lower values (in particular, Gof $<5$ ) indicate a better quality of fit for the global

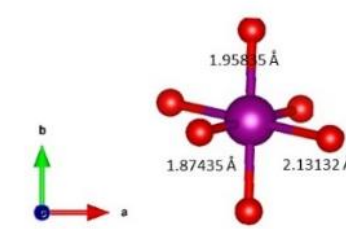

LSMO25MW Pnma

$157^{\circ}$

Mn-O-Mn along $b$ Mn-O-Mn along a $154^{\circ}$

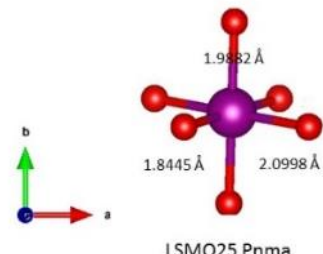

LSMO25 Pnma

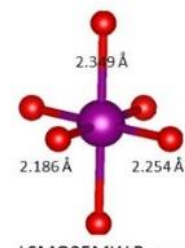

LSM035MW Pnma

$155^{\circ}$

$154^{\circ}$

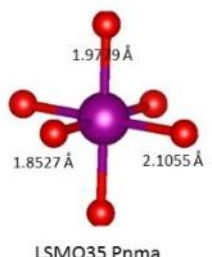

$157^{\circ}$

$154^{\circ}$

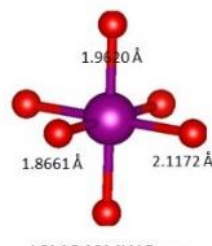

LSMO40MW Pnma

$157^{\circ}$

$154^{\circ}$

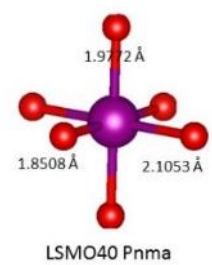

$157^{\circ}$

$154^{\circ}$
Mn-O-Mn along a $\quad 154^{\circ}$

Figure 5 - Mn-O polyhedron for the LSMO samples LSMO-Con and LSMO-MW samples $(x=0.25,0.35$ and 0.4$)$ highlighting the bond lengths and $\mathrm{Mn}^{3+}-\mathrm{O}-\mathrm{Mn}^{4+}$ bond angles as an illustration of the extent of Jahn-Teller distortion with increasing
strontium dopant

data set. The Gof of LSMO samples ( $x=0.25,0.35$ and 0.4 ) synthesised using MW irradiation is much lower than the conventionally synthesised samples, indicating that the global refinement was more successful for the MW samples. However, looking at the reliability markers for the individual phases (i.e. $R_{\text {bragg }}$ values) contained in the polycrystalline sample, we see much greater values for the Pnma phases of LSMO synthesised using MW irradiation compared to the Pnma phases of the conventionally synthesised LSMO. This high $\mathrm{R}_{\text {bragg }}$ value would infer that the Pnma phase present in the MW sample is not as adequately represented by the model structure in spite of the improved quality of the fit for the entire data set (low Gof). The slight inadequacies in the model parameters for the Pnma phase in the MW samples are evident in the Fourier difference maps shown in Table 3 which have been generated for each crystalline phase in each sample. ${ }^{63}$ These maps are shown as isosurfaces with positive and negative electron density. ${ }^{64}$ Positive electron density in a Fourier difference map indicates that a structure is observed where there is no structure in the model, and negative density indicates that a structure is not observed where there is a structure in the model. ${ }^{64}$ The green surfaces (no difference in electron density) in the maps show for the rhombohedral (R-3c) phases in Table 3 show that this polymorph has been well characterised by the model parameters (Table 2) used in the Rietveld refinement i.e. the model and observed structures are in good agreement. However the colour variation in maps for the orthorhombic (Pnma) phases indicates that this polymorph has not been as well characterised by the model parameters. ${ }^{63}$

The increasing polarisation in electron density values for the Pnma phases in the MW-assisted synthesised materials compared with the conventionally synthesised materials is 


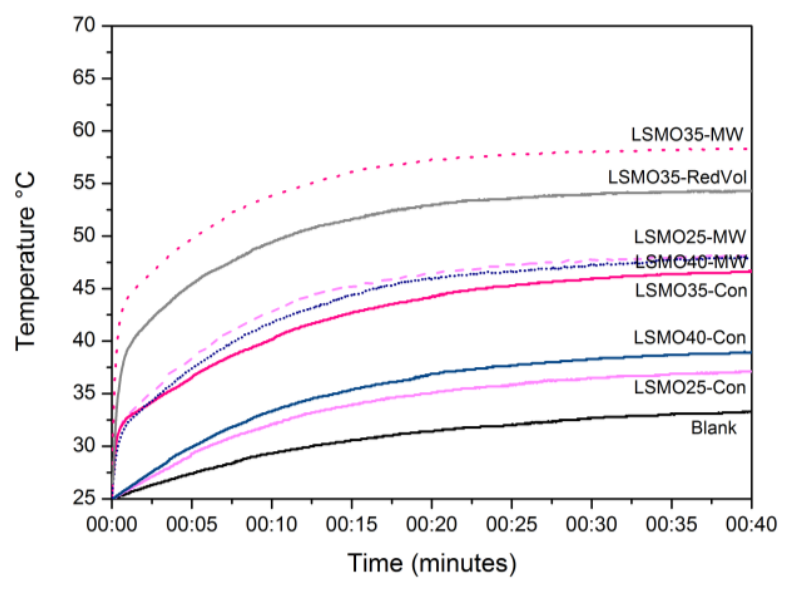

Figure 6 - Heating Curves of $10 \mathrm{mg} \mathrm{mL}^{-1}$ aqueous suspensions of the LSMO samples (a) shows the comparison between the conventionally synthesised 32 LSMO25-Con,
LSMO35-Con and LSMO40-Con (solid lines) with the MW-assisted synthesis of each (broken lines) (LSMO25-MW, LSMO35-MW and LSMO40-MW. The grey dashed line shows the conventional synthesised LSMO with water omitted (LSMO35-RedVol) as a better comparison for LSMO35-MW.

evidence of discrepancies between the calculated model and observed reflections. ${ }^{65}$ Unsuccessful attempts were made to improve the model further, by altering the atomic co-ordinates based on the presence and absence of atoms in the Fourier difference maps. The data shown in Table 2 is from the best refinement. A possible cause of the discrepancies between the calculated model and observed reflections evident in the Fourier difference maps for the Pnma structures synthesised by different methods is the initial rapid heating associated with MW. Not only may this have led to the more discrete nanoparticles (low Gof), but the rapid heating may also have led to the formation of the less stable orthorhombic (Pnma) crystal phases shown here, which are not as well represented by the model (high $R_{\text {Bragg }}$ vaue). This may have consequences on the magnetic properties of the LSMO synthesised using MWirradiation, where the less stable Pnma polymorphs may be more susceptible to a change in the applied magnetic field vide infra and produce an enhanced MCE.

Figure 5 shows the $\mathrm{Mn}-\mathrm{O}$ octahedrons present in LSMO responsible for the magnetic properties of the materials which have been isolated using the VESTA software following Rietveld analysis of the powder diffraction patterns. ${ }^{58}$ On comparing the octahedrons of the LSMO samples synthesised using MW irradiation with those synthesised using conventional heating, there appears to be a substantial increase in the average $\mathrm{Mn}-\mathrm{O}$ bond length for LSMO35-MW, but little difference for LSMO25MW and LSMO40-MW. In addition, on comparison of the standard deviations of the bond lengths, there appears to be an overall reduction in the variations of the $\mathrm{Mn}-\mathrm{O}$ bond lengths with increasing strontium content. This reduction in the variation of bond lengths as a function of strontium dopant is to be expected as increasing $\mathrm{Sr}^{2+}$ induces an increase in the proportion of $\mathrm{Mn}^{4+}$ and a simultaneous reduction in the amount of the Jahn-Teller active $\mathrm{Mn}^{3+}$ ions. ${ }^{66-68}$ Jahn-Teller distortion (elongation or contraction of bond lengths) leads to a lack of degeneracy in either the $t_{2 g}$ or $e_{g}$ orbitals which is necessary for the material to exhibit magnetic properties via the doubleexchange mechanism where one 'spin up' electron is transferred from the intermediate oxide to the $\mathrm{Mn}^{4+}$, and is replaced by another electron from the high-spin $\mathrm{Mn}^{3+} .{ }^{23}$

As previously mentioned, when comparing the bond lengths of samples synthesised using the two different types of syntheses, LSMO35-MW shows an unexpected reduction of $35 \%$ in the variation of the bond lengths compared with LSMO35-Con. This would typically be associated with a reduction in Jahn-Teller distortion and a reduction in magnetic properties in 3.1.2, which is not seen. The reduction in the variation of bond lengths and increase in the average $\mathrm{Mn}-\mathrm{O}$ bond length for LSMO35-MW leads to the existence of a much larger unit cell volume (327.3 $\AA^{3}$ ) as shown in Table 2 , which also forms the smallest aggregate crystal size for any of the LSMO materials (as seen in S.I. Figure S1). The smaller aggregates in LSMO35-MW may better participate in Brownian and Néel relaxations, along with greater heat loss through magnetic hysteresis. Thus aggregate crystal size may be a more influential parameter compared to $\mathrm{Mn}-\mathrm{O}$ bond lengths and variations in determining the magnetic properties of the material.

In addition to the anomalous average and variation of $\mathrm{Mn}$ $\mathrm{O}$ bond lengths for LSMO35-MW, there is also an inconsistency in the $\mathrm{Mn}-\mathrm{O}-\mathrm{Mn}$ bond angle along the $\mathrm{b}-$ axis for LSMO35-MW, where we see a slight decrease of $2^{\circ}$ compared with the other samples. This reduction in the size of the bond angle ( $\mathrm{Mn}-\mathrm{O}-\mathrm{Mn}$ ) coincides with the increasing length of the $b$ - side of the unit cell (Table 2) for the material. The increasing average bond length and decreasing $\mathrm{Mn}-\mathrm{O}-\mathrm{Mn}$ bond angle may together indicate greater tiling of the $\mathrm{Mn}$-octahedra, and possibly encourage longer-range ferromagnetic order to be determined in the magnetic heating experiments vide infra. ${ }^{68}$ The increasing range of ferromagnetic order in LSMO35-MW caused by the tilting of the $\mathrm{Mn}$-octahedra means that the anomalous bond lengths and angles for this material may be justified.

\subsubsection{Magnetic Heating Experiments}

The magnetic properties of the LSMO materials $(x=0.25,0.35$ and 0.4 ) synthesised using this new MW-assisted method were preliminarily characterised using induction heating experiments for LSMO samples in order to confirm the most suitable stoichiometry of LSMO for use in MFH. The magnetic heating properties of LSMO at a range of dopants $(x=0.25,0.35$ and 0.4$)$ were improved using this $\mathrm{MW}$-assisted peroxide sol-gel method when compared to conventional heating in the peroxide sol-gel method used in a previous work. ${ }^{32}$ Figure 6 draws comparison between the two syntheses; using conventional heating or MWassisted heating. As previously discussed, it was necessary to omit the water from the modified-peroxide sol-gel synthesis and use only the excess alkaline hydrogen peroxide in order to maximise the amount of product able to be synthesised in a 10 $\mathrm{mL}$ microwave tube. In order to make a comparison between the conventional ${ }^{32}$ and $\mathrm{MW}$-assisted syntheses we can look at LSMO35-RedVol. This is $\mathrm{La}_{0.65} \mathrm{Sr}_{0.35} \mathrm{MnO}_{3}$ which has been heated in a conventional manner, but without additional water. LSMO35-RedVol displays an improved MCE compared with the conventional synthesis (LSMO35-Con), but the magnetic heating properties are further enhanced when the synthetic 

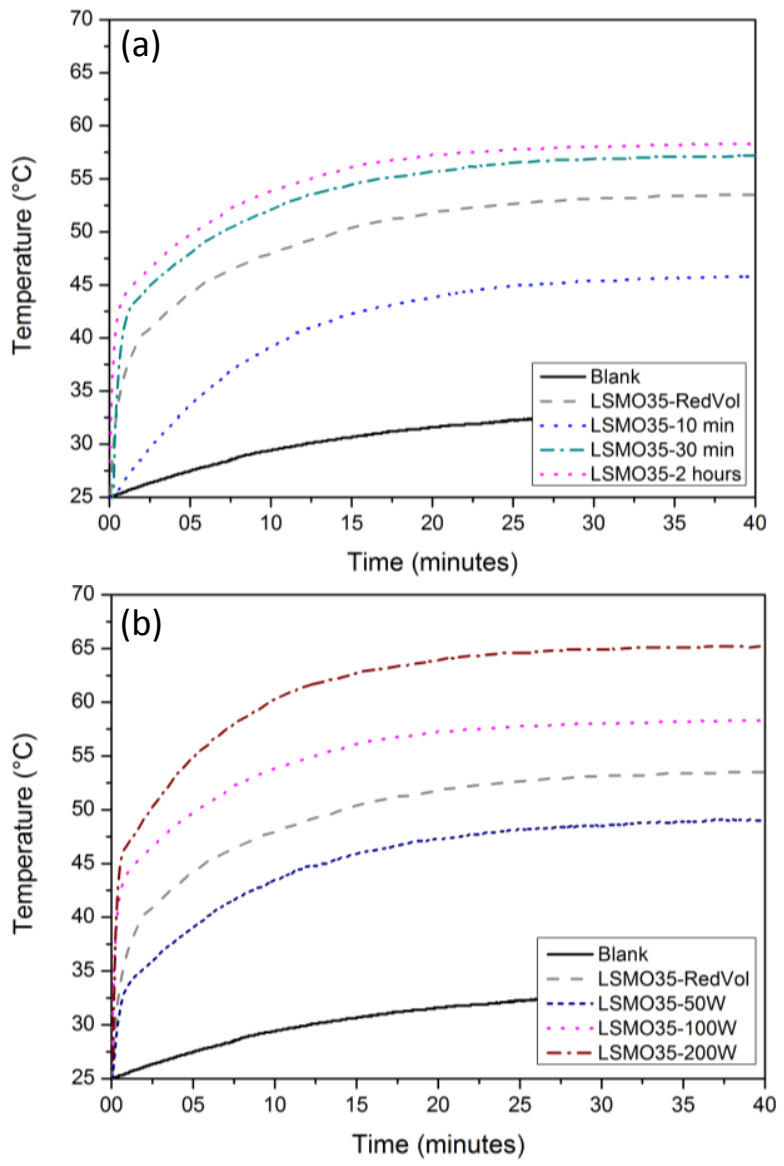

Figure 7 - (a) shows the comparison of LSMO35 samples synthesised for differen time intervals ( 10 minutes, 30 minutes and 2 hours) at $100 \mathrm{~W}$ compared with the conventional method of heating with water omitted (LSMO35-RedVol), and (b) shows the comparison of LSMO35 samples
settings $(50,100$ and $200 \mathrm{~W})$ for 2 hours.

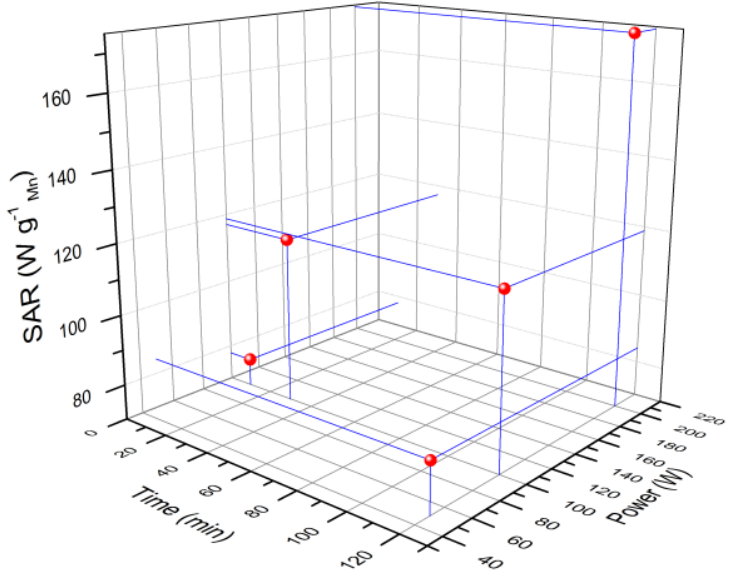

Figure 8 -SARs of LSMO35 samples synthesised for different time intervals (10 minutes, 30 minutes and 2 hours) at $100 \mathrm{~W}$ and various MW power settings (50, 100 and $200 \mathrm{~W}$ ) for 2 hours.

method included heating with $\mathrm{MW}$-assistance shown by LSMO35-MW. The heating trend remains consistent between the conventional and $\mathrm{MW}$-assisted syntheses with $\mathrm{La}_{0.65} \mathrm{Sr}_{0.35} \mathrm{MnO}_{3}$ being the optimal dopant for superior MCE, thus reinforcing our intention to improve the physical properties of this material. The improvement in crystallinity seen with the reduction in the volume of the reactant mixture between LSMO35-Con and LSMO35-RedVol may be due to a more concentrated 'sol' favouring the precipitation of smaller and more discrete nanoparticles. The more discrete and crystalline the MNPs as previously mentioned was thought to lead to greater ferromagnetic core contributions. ${ }^{62}$ This is confirmed in Figure 6 where we see an enhancement of MCE from LSMO35-Con to LSMO35-MW. The rest of this investigation (Section 3.2) deals with the study of the magnetic properties of LSMO where $x=0.35$ synthesised at a range of power and time settings with the aim of optimising the MCE for the purposes of MFH.

\subsection{Optimising Structural and Magnetic Properties of LSMO35 using $\mathrm{MW}$ irradiation}

\subsubsection{Magnetic heating Experiments}

In Section 3.1, the most suitable stoichiometry of LSMO synthesised using the MW-assisted peroxide sol-gel synthesis for use in MFH was shown to be $\mathrm{La}_{0.65} \mathrm{Sr}_{0.35} \mathrm{MnO}_{3}$ in keeping with prior work. ${ }^{32}$ Due to the comparable orthorhombic and rhombohedral crystal structure distributions for the MW and conventionally synthesised samples of LSMO35, we would have expected to see extremely similar physical properties such as the MCE investigated through the induction heating experiments and magnetic hysteresis. However the differences in physical properties between the samples (magnetic susceptibility, hysteresis and magnetocaloric effect) presented cannot be explained purely in terms of crystal structure composition (Pnma or R-3c), but instead the degree of crystallinity. In this section, we aim to optimise the MCE produced by LSMO35-MW by adjusting the MW parameters (time and power) and investigating the effect on physical properties through magnetic heating experiments and SQUID magnetometry.

Figures 7 (a) and (b) show the heating curves for $10 \mathrm{mg} \mathrm{mL}$ 1 aqueous suspensions of LSMO samples in order to illustrate the MCEs of the various materials. In Figure 7 a) we can see that samples synthesised using MW heating for 30 min (LSMO35-30 $\mathrm{min}$ ) and 2 hours (LSMO35-2hr) follow a similar heating pattern as when conventional heating (LSMO35-Con) is used in the synthesis. For these samples a rapid monotonic increase in temperature is initially recorded for all samples followed by saturation. From previous work we were able to see that such a rapid initial increase in temperature appears to be specific to LSMO35. ${ }^{32}$ As previously mentioned, this section is dealing with the optimisation of the $\mathrm{MW}$ parameters used to synthesise LSMO35, and as a result detailed structural analysis was not conducted on LSMO35 samples synthesised for different time intervals (LSMO35-10min and LSMO-30min) or for various power settings (LSMO35-50W and LSMO35-200W). Due to the absence of this rapid initial increase in temperature we can suggest that the MW synthesis time of 10 minutes was not sufficient for the cations to diffuse within the lattice to produce the perovskite LSMO35. ${ }^{61}$ As the time in which the samples were synthesised under MW irradiation increases from $10 \mathrm{~min}$ to $30 \mathrm{~min}$ to 2 hours, we see an increase in the saturation 


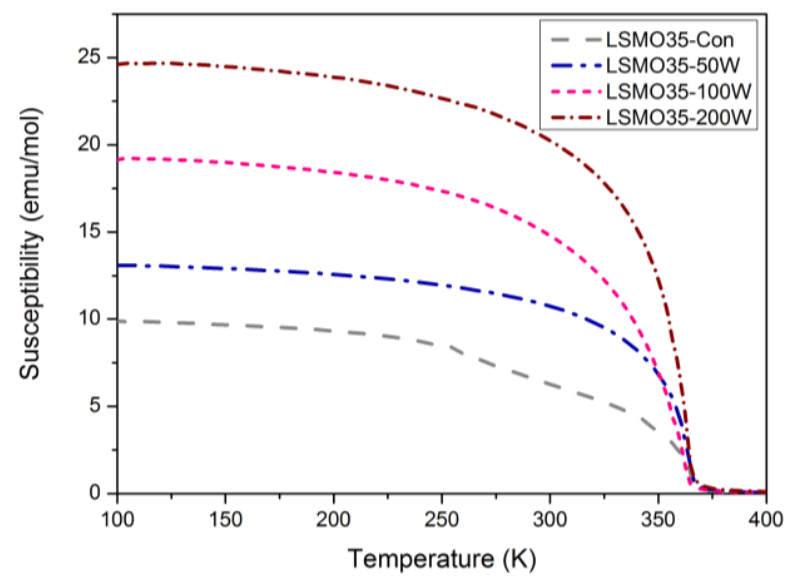

Figure 9 - Magnetic susceptibilities measurements conducted under Zero-Field Cooled (ZFC) conditions of LSMO35 samples synthesised through conventiona heating (LSMO35-Con) ${ }^{32}$ and MW heating at various power settings (50 W, 100
and $200 \mathrm{~W}$ ) for 2 hours (LSMO35-50W, LSMO35-100W and LSMO35-200W).

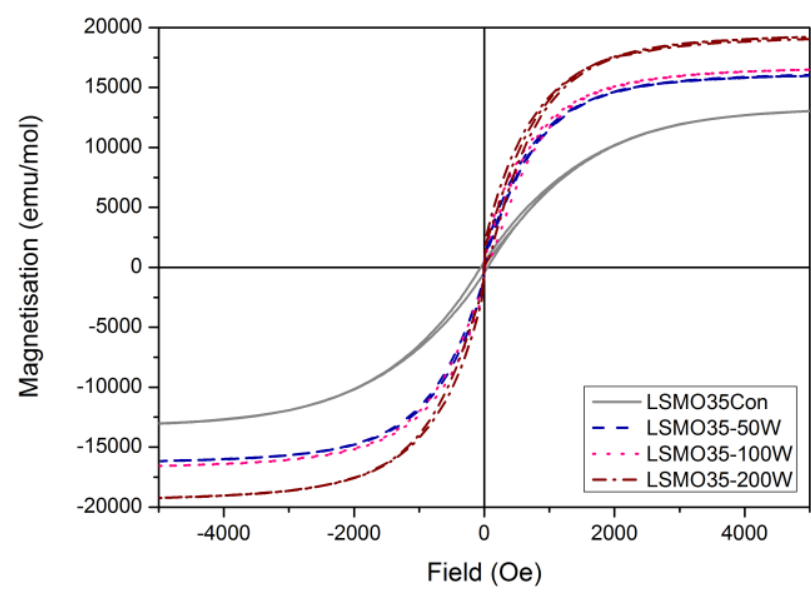

Figure 10 - Hysteresis loops measured at $100 \mathrm{~K}$ for LSMO35 samples synthesised through conventional heating (LSMO35-Con) from a previous work ${ }^{32}$ and $\mathrm{MW}$ heating at various power settings ( $50 \mathrm{~W}, 100 \mathrm{~W}$ and $200 \mathrm{~W}$ ) for 2 hours (LSMO35-
$50 \mathrm{~W}$, LSMO35-100W and LSMO35-200W). The complete field range measured is shown (-5000 to 5000 Oe)

Table 4 - Parametric characterisation of hysteresis for a selection of doped LSMO35 materials (LSMO35-Con, LSMO35-50W, LSMO35-100W and LSM035-200W). ( $\mathrm{M}_{\mathrm{s}}$ - Saturation Magnetisation and $\mathrm{T}_{\mathrm{c}}$ - Curie temperature)

\begin{tabular}{ccc}
\hline Sample & $\mathrm{M}_{\mathrm{s}}$ & $\mathrm{T}_{\mathrm{c}}$ \\
& emu/mol & $\mathrm{K}$ \\
\hline LSM035-Con & 13,000 & 362 \\
LSM035-50W & 16,000 & 360 \\
LSM035-100W & 17,000 & 360 \\
LSM035-200W & 19,000 & 360 \\
\hline
\end{tabular}

temperatures of the samples from $45.9^{\circ} \mathrm{C}$ to $57.0^{\circ} \mathrm{C}$ to $58.3^{\circ} \mathrm{C}$ respectively. The samples synthesised using MW heating for 30 minutes and 2 hours were both found to exhibit superior MCEs in the induction heating experiments compared with the conventional synthesis; heating in the linear regime for longer, and obtaining higher saturation temperatures. Figure 7 (a) therefore shows that MW heating leads to a fourfold decrease in the reaction time needed for the synthesis of LSMO35 whilst at the same time, producing materials with superior MCE.

Figure 7 (b) shows the effect of changing the MW power from 50 to 100 to $200 \mathrm{~W}$ against the conventional method of heating, on the magnetic heating properties of the material. The heating curves are shown for samples synthesised using the MW for the same length of time as the conventional synthesis (2 hours) LSMO35 samples synthesised using MW irradiation for 2 hours at 100 and $200 \mathrm{~W}$ show a rapid increase in temperature compared with the conventional synthesis. We can see that the samples synthesised using the MW synthesis at both 100 and $200 \mathrm{~W}$ heat in the linear regime for longer than the sample synthesised using conventional heating, in addition to reaching greater saturation temperatures. The saturation temperature in the magnetic heating measurements appears to be a function of the MW power used in the synthesis, with the $200 \mathrm{~W}$ setting showing superior heating results as a maximal temperature of $65.1^{\circ} \mathrm{C}$ is reached compared with $58.3^{\circ} \mathrm{C}$ using $100 \mathrm{~W}$ and 49.1 ${ }^{\circ} \mathrm{C}$ with $50 \mathrm{~W}$ MW power.

Figure 8 characterises the improvement in magnetic heating properties in terms of the SAR, where we see the optimal SAR of $175 \mathrm{Wg}^{-1} \mathrm{Mn}$ was achieved using the MW synthesis for 2 hours at $200 \mathrm{~W}$. This far exceeds the conventional maximum of 56 $\mathrm{Wg}^{-1} \mathrm{Mn}$. The substantial difference in the magnetic heating properties of the LSMO35 samples synthesised at various power settings may lie with the evaporation step, where the water produced by the decomposition of hydrogen peroxide is removed by heating to $110{ }^{\circ} \mathrm{C}$. The rate at which this happens varies; taking longer at lower power settings. For example, this stage takes 30 minutes using $50 \mathrm{~W}$, whereas it only takes 15 minutes when $200 \mathrm{~W}$ is used.

\subsubsection{Magnetic measurements}

Figure 9 shows the magnetic susceptibility comparison for our LSMO35 samples synthesised using MW irradiation at various power settings ( $50 \mathrm{~W}, 100 \mathrm{~W}$ and $200 \mathrm{~W}$ ) compared with conventional heating. The magnetic susceptibilities of all of the MW samples are larger than the sample synthesised using conventional heating, and there are also much steeper transitions for these $\mathrm{MW}$ samples. Comparing the magnetic susceptibilities of the MW samples at low temperatures, they are shown to increase as a function of increasing power setting (50 W, $100 \mathrm{~W}$ and $200 \mathrm{~W}$ ) used to synthesise LSMO35. We attribute this in part to the higher power setting evaporating the water from the sol-gel mixture to produce better crystallised nanoparticles which will have thinner non-magnetic surface layers and fewer crystal defects.

The greater magnetic volume of the better crystallised nanoparticles (LSMO35-MW/LSMO35-100W) seen in the SEM synthesis comparison (Figure 4) would have meant that a greater proportion of the material able to participate in the double exchange mechanism and lead to enhanced magnetic properties such as was seen in the magnetic heating experiments in Section 3.2.1. This supports Hong et al.'s observation of an increase in magnetic susceptibility (4.5 emu $\mathrm{g}^{-1}$ ) when $\mathrm{Fe}_{3} \mathrm{O}_{4}$ was synthesised using $\mathrm{MW}$ irradiation as opposed to conventional heating. ${ }^{69}$ They attributed this 
enhancement in magnetic susceptibility to better crystallised nanoparticles, pointing out that in that instance there was little difference in the average crystallite size $(8-9 \mathrm{~nm})^{69}$, unlike in this case where there is a significant difference in the average crystallite size (Table 2) decreasing from $24.8 \mathrm{~nm}$ for LSMO35Con to $8.8 \mathrm{~nm}$ for the orthorhombic (Pnma) polymorph of LSMO35-MW for example.

In investigations on $\mathrm{La}_{0.7} \mathrm{Sr}_{0.3} \mathrm{MnO}_{3}{ }^{70}$ and $\mathrm{La}_{0.8} \mathrm{Sr}_{0.2} \mathrm{MnO}_{3}{ }^{71}$ the magnetisation of the nanoparticles was thought to be strikingly dependent on the particle size. The rationale behind this was based on a model by Zhang et al. which suggested that larger magnetic particles would have a smaller surface area to volume ratio, meaning that they would have a larger ferromagnetic core compared with anti-ferromagnetic "magnetically dead layer", and hence would exhibit a larger magnetic response than a smaller magnetic particle. ${ }^{35}$ The driving force for this, Kameli et al. suggest, is microstrain. ${ }^{71}$ In this work we see a reduction of $33 \%$ in crystallite diameter (Table 2) when the MW-assisted synthesis is used (LSMO35$100 \mathrm{~W}$ ), compared with conventional heating (LSMO35-Con). With this drop in crystallite size, one would expect an associated drop in magnetic susceptibility as the ferromagnetic core volume would decrease if only Zhang's model is considered. ${ }^{35}$ However, MW conditions appear to have improved LSMO35 crystallinity, and in turn reduced the magnetically dead layer, which was calculated for LSMO35-Con and LSMO35-MW to be $6.11 \mathrm{~nm}$ and $2.12 \mathrm{~nm}$ respectively (for respective average crystallite sizes of $82.4 \mathrm{~nm}$ and $61.8 \mathrm{~nm}) .{ }^{72}$ Thus the increase of approximately $60 \%$ in the zero-field cooled magnetic susceptibility (between LSMO35-Con and LSMO35-MW) shown in Figure 9 can be rationalised by increasing ferromagnetic contribution associated with the improvement in crystallinity, but also suggests reduced cluster formation and greater ordering of magnetic domains also. ${ }^{6}$ This was previously suggested by Epherre et. al who proposed that the reduced proportion of magnetic clusters (as also seen in Section 3.1 in this work), could lead to fewer local distortions within the particle cores, in turn increasing the saturation magnetisation $\left(M_{s}\right)$ (and magnetic susceptibility) which is seen in this work. ${ }^{6}$

Figure 9 also shows that all samples undergo a ferromagnetic to paramagnetic transition with little difference in the transition temperature $(2 \mathrm{~K})$ with $\mathrm{T}_{\mathrm{c}}$ 's of $362 \mathrm{~K}$ for LSMO35-Con and $360 \mathrm{~K}$ (reported in Table 4) for all of the samples synthesised using MW-irradiation (LSMO35-50W, LSMO35-100W and LSMO35-200W). T's 's were obtained using the Arott plot method, where the inverse of magnetic susceptibility is plotted against the square of magnetisation with respect to temperature (Supplementary Figure S12). This is in keeping with the method of calculation of $T_{c}$ used in a previous work which was useful for the LSMO35-Con sample. ${ }^{32}$ The similar $T_{c}$ 's for LSMO35 samples indicates that the ferromagnetic phase contribution to magnetisation is similar. This also indicates that the stoichiometry and crystal structure are similar. However the slight inflections at around $310 \mathrm{~K}$ and $250 \mathrm{~K}$ for LSMO35-Con are indicative of minor phases which have differing stoichiometries. These minor phases were not however quantifiable using Rietveld analysis of the powder diffraction patterns. Of course this could be due to the surface phase, which according to Zhang's model suggests will have a lower transition temperature and distinct magnetic characteristics such as coercivity. ${ }^{35}$ The magnetic hysteresis loops in Figure 10 show that the LSMO35-200W sample appears to be a harder ferromagnet, with increasing coercivity and remanence compared to LSMO synthesised at lower MW power settings ( 50 and $100 \mathrm{~W}$ ) or using the conventional heating method. The increasing coercivity between the conventional and MW samples is also in keeping with the decreasing crystallite size of the orthorhombic (Pnma) phase shown in Table 2. The static field conditions used for the magnetic measurements however cannot be used to infer the hysteretic contribution to the magnetocaloric effect under alternating magnetic field conditions.

Comparison of the magnetic susceptibility and magnetic saturation values with literature values is difficult in this instance. To the knowledge of the authors there are no magnetic characterisation studies conducted at the dopant level where $x=0.35$ with similar measurement parameters (field dependent magnetisation measurements in the range $-10,000 \leq \mathrm{H} \leq 10,000 \mathrm{Oe}$, at $100 \mathrm{~K}$ and $200 \mathrm{~K}$, with susceptibility measurements in field cooled (FC) and zero field cooled (ZFC) conditions between 100 and $370 \mathrm{~K}$, at $500 \mathrm{Oe}$.). For example, Manh et al. conducted these magnetic saturation measurements at a range of temperatures but only at $100 \mathrm{Oe}$ for LSMO where $\mathrm{x}=0.3 .{ }^{73}$ Kačenka et al. conducted magnetic susceptibility measurements at $7.96 \mathrm{kA} / \mathrm{m}$ ( 1206 Oe) and magnetic saturation measurements between -200 and 200 Oe at $4.5 \mathrm{~K}$ for LSMO where $\mathrm{x}=0.35^{74}$ and Rostamnejadi et al. used an applied magnetic field of $293 \mathrm{~K}$ between $5,000 \leq \mathrm{H} \leq 5,000$ Oe for LSMO where $\mathrm{x}=0.33 .75$

Theoretically the optimal magnetocaloric effect can be observed in the region of the $T_{c}$. Comparing the $T_{c}$ 'S of LSMO35Con and the MW samples (LSMO35-100W and LSMO35-200W) with the magnetic heating experiments we can see that the magnetic heating regime for the MW samples are much closer to their $T_{c}{ }^{\prime}$ s. For example, the maximum temperature in the magnetic heating experiments reached by LSMO35-100W sample is only $28.7 \mathrm{~K}$ below the $T_{c}$, whereas there is a difference of $42.3 \mathrm{~K}$ between $\mathrm{T}_{\max }$ and $\mathrm{T}_{\mathrm{c}}$ for the LSMO35-Con sample. The ferromagnetic to paramagnetic transition gradient of LSMO35Con is -0.2 emu mol-1 $\mathrm{K}^{-1}$, compared with -0.6 emu $\mathrm{mol}^{-1} \mathrm{~K}^{-1}$ for LSMO35-50W and LSMO35-100W, and -0.8 emu $\mathrm{mol}^{-1} \mathrm{~K}^{-1}$ for LSMO35-200W. LSMO35-200W not only has a larger magnetic susceptibility compared to the other samples, but its larger transition gradient means that it will have ferromagnetic character for longer with increasing temperatures until its $T_{c}$ where there is a sudden drop in magnetic susceptibility. In contrast, LSMO35-Con has a weak transition gradient, meaning that its ferromagnetic character weakens further in advance of its $T_{c}$.

Figure 11 demonstrates the improvement in magnetic susceptibility with increasing $\mathrm{MW}$ power settings during synthesis. This coincides with an observed improvement in the crystallinity of the samples in the micrographs in the Supplementary Information Figure S11. The improved 
magnetocaloric effect (SAR) observed for the $M W$ samples (LSM035-50W, LSMO35-100W and LSM035-200W) compared to the conventionally synthesised sample (LSMO35-Con) can therefore be ascribed to the greater magnetic susceptibility (shown in Figure 11), in addition to the sharper ferromagnetic to paramagnetic transition as previously mentioned. ${ }^{29}$ These attributes will mean faster, more efficient switching of the magnetic dipoles within the LSMO35-MW sample with an alternating magnetic field, which will generate more heat in the process via Néel and Brownian rotations. ${ }^{76}$

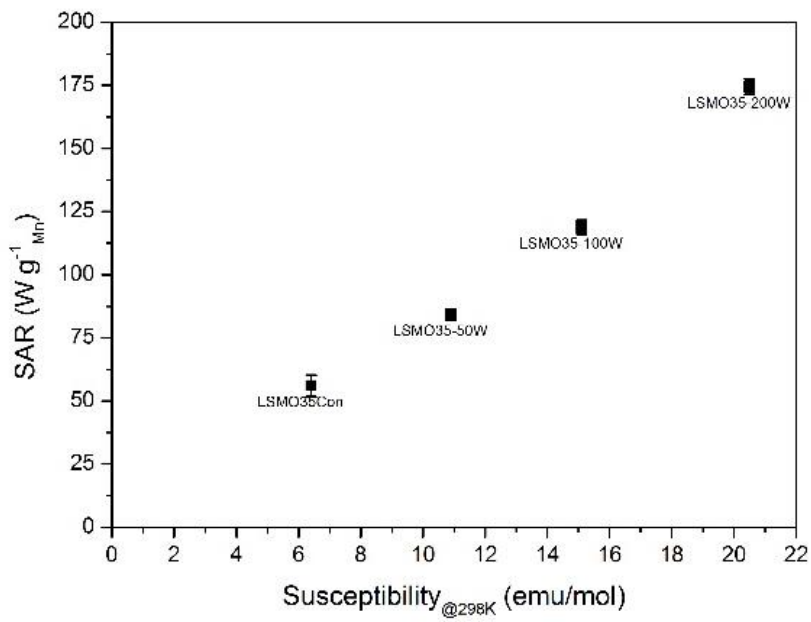

Figure 11 - The relationship between magnetic susceptibility and the magnetocaloric effect of $\mathrm{La}_{0.65} \mathrm{Sr}_{0} \mathrm{MnO}_{3}$ (LSMO35) synthesised at various power LSMO35. ${ }^{32}$ The magnetocaloric effect is measured through magnetic heating experiments and characterised using the Specific Absorption Rate (SAR). Th magnetic susceptibility values used are the Zero-field cooled measurements at
$298 \mathrm{~K}$.

\section{Conclusions}

The use of MW irradiation was shown in this work to improve the crystallinity of LSMO at a range of dopants $(x=0.25,0.35$ and 0.4 ) synthesised using a modified peroxide sol-gel synthesis. In spite of the new synthetic MW-assisted method used and the resulting change in the degree of crystallinity of the product, there was no significant change in proportions of orthorhombic to rhombohedral crystal geometries present. The magnetocaloric effect of each material was observed through induction heating experiments and $\mathrm{La}_{0.65} \mathrm{Sr}_{0.35} \mathrm{MnO}_{3}$ (LSMO35) proved to have the optimal dopant concentration as was shown in the conventional synthesis. ${ }^{32}$ Through attempts to optimise the magnetic properties of LSMO35, we were also able to show a linear relationship between the MW power settings used (50, 100 and $200 \mathrm{~W}$ ) and the magnetocaloric effect (producing SARs of $84.2,118.8$ and $174.4 \mathrm{~W} \mathrm{~g}^{-1} \mathrm{Mn}$ respectively) indicating the importance of the evaporation step in the $\mathrm{MW}$-assisted peroxide sol-gel synthesis. This work shows the advantageous use of MW irradiation in material synthesis to enhance not only crystallinity, but also magnetic properties (magnetic susceptibility, magnetocaloric effect and magnetic hysteresis) through a greater core ferromagnetic contribution and also greater magnetic ordering. ${ }^{35}$ The more discrete crystalline LSMO35 materials synthesised in this work using MW irradiation (at 100 and $200 \mathrm{~W}$ for more than 30 minutes) will be more effective mediators for MFH compared to the conventionally synthesised material, through their ability to produce an enhanced MCE and also due to the increased degree of crystallinity meaning that the agglomerate particles will be better broken down by milling techniques to the desired agglomerate size of the order of $100 \mathrm{~nm}$.

\section{Acknowledgements}

Katherine McBride, Suzanne Bennington-Gray and James Cook would like to acknowledge financial support from the Department of Education and Learning Northern Ireland (DEL$\mathrm{NI}$ ) and Engineering and Physical Sciences Research Council (ESPRC) respectively for PhD studentships. The authors also acknowledge the help of Professor David Rooney and Christopher McCallum for their help with the induction heating experiments.

\section{Notes and references}

1 Y. Jiang, J. Deng, S. Xie, H. Yang and H. Dai, Ind Eng Chem Res, 2015, 54, 900-910 (DOI:10.1021/ie504304u).

2 M. Romero, H. Pardo, R. Faccio, M. A. Tumelero, C. C. P. Cid, J. Castiglioni, A. A. Pasa and Á W. Mombrú, J Magn Magn Mater, 2015, 382, 342-348.

3 Z. Y. Zhou, G. S. Luo and F. Y. Jiang, J Magn Magn Mater, 2009, 321, 1919-1923.

4 S. Vasseur, E. Duguet, J. Portier, G. Goglio, S. Mornet, E. Hadová, K. Knížek, M. Maryško, P. Veverka and E. Pollert, J Magn Magn Mater, 2006, 302, 315-320.

5 S. Jadhav, D. Nikam, V. Khot, N. Thorat, M. Phadatare, R. Ningthoujam, A. Salunkhe and S. Pawar, New Journal of Chemistry, 2013, 37, 3121-3130.

6 R. Epherre, C. Pepin, N. Penin, E. Duguet, S. Mornet, E. Pollert and G. Goglio, Journal of Materials Chemistry, 2011, 21, 14990-14998.

7 O. Kaman, P. Veverka, Z. Jirák, M. Maryško, K. Knižek, M. Veverka, P. Kašpar, M. Burian, V. Šepelák and E. Pollert, Journal of Nanoparticle Research, 2011, 13, 1237-1252.

8 E. Pollert, K. Knížek, M. Maryško, P. Kašpar, S. Vasseur and E. Duguet, J Magn Magn Mater, 2007, 316, 122-125).

9 F. K. Storm, W. Harrison, R. S. Elliott, L. R. Kaiser, A. W. Silberman and D. L. and Morton, J. Microw. Power, 1981, 16, 179-184.

10 S. Mornet, S. Vasseur, F. Grasset, P. Veverka, G. Goglio, A. Demourgues, J. Portier, E. Pollert and E. Duguet, Progress in Solid State Chemistry, 2006, 34, 237-247.

11 V. Pecharsky and K. and Gschneidner Jr, J Magn Magn Mater, 1999, 200, 44-56.

12 J. R. Gómez, R. F. Garcia, A. D. M. Catoira and M. R. and Gómez, Renewable and Sustainable Energy Reviews, 2013, 17, 74-82.

13 M. Phan and S. and Yu, J Magn Magn Mater, 2007, 308, 325-340. 14 S. B. Roy, in Handbook of Magnetic Materials, ed. K. H. J. Buschow, Elsevier, 2003, p. 203-316.

15 Y. Fukumori and H. and Ichikawa, Advanced Powder Technology, 2006, 17, 1-28.

16 O. A. Kuznetsov, O. N. Sorokina, V. G. Leontiev, O. A. Shlyakhtin, A. L. Kovarski and A. A. Kuznetsov, J Magn Magn Mater, 2007, 311, 204-207. 
17 R. M. Ferguson, K. R. Minard and K. M. Krishnan, J Magn Magn Mater, 2009, 321, 1548-1551.

18 I. Brigger, C. Dubernet and P. Couvreur, Adv. Drug Deliv. Rev., 2002, 54, 631-651.

19 V. Uskoković, A. Košak and M. Drofenik, Mater Lett, 2006, 60, 2620-2622.

20 X. Zhu, Perovskite nanopowders: Synthesis, characterization, properties and applications, 2011.

21 Q. Huang, A. Santoro, J. Lynn, R. Erwin, J. Borchers, J. Peng and R. Greene, Physical Review B, 1997, 55, 14987-14999.

22 S. Majumdar and S. van Dijken, J. Phys. D, 2013, 47, 034010034025.

23 C. Zener, Physical Review, 1951, 82, 403-405.

24 P. W. Anderson and H. Hasegawa, Physical Review, 1955, 100, 675-681.

25 P. De Gennes, Physical Review, 1960, 118, 141-154.

26 S. O. Manjunatha, A. Rao, T. Lin, C. Chang and Y. Kuo, J. Alloys Compounds, 2015, 619, 303-310.

27 A. Millis, P. Littlewood and B. I. Shraiman, Phys. Rev. Lett., 1995 74, 5144-5147.

28 V. Markovich, I. Fita, D. Mogilyansky, A. Wisniewski, R. Puzniak, L. Titelman, L. Vradman, M. Herskowitz and G. Gorodetsky, Journal of Physics Condensed Matter, 2007, 19, 346210-10pp.

29 V. Markovich, A. Wisniewski and H. Szymczak, in Handbook of Magnetic Materials, ed. K. H. J. Buschow, Elsevier B.V, Great Britain, 2014, p. 1-201.

30 P. Žvátora, M. Veverka, P. Veverka, K. Knížek, E. Pollert, V. Král, G. Goglio, E. Duguet, K. Záveta and O. Kaman, Journal of Solid State Chemistry, 2013, 204, 373-379.

31 E. Natividad, M. Castro, G. Goglio, I. Andreu, R. Epherre, E. Duguet and A. Mediano, Nanoscale, 2012, 4, 3954-3962 (DOI:10.1039/C2NR30667K).

32 K. McBride, J. Cook, S. Gray, S. Felton, L. Stella and D. Poulidi, CrystEngComm, 2016, 18, 407-416.

33 A. Gaur and G. D. Varma, Journal of Physics: Condensed Matter, 2006, 18, 8837-8846.

34 L. da Conceição, C. R. Silva, N. F. Ribeiro and M. M. Souza, Mater Charact, 2009, 60, 1417-1423.

35 N. Zhang, W. Ding, W. Zhong, D. Xing and Y. Du, Physical Review $B, 1997,56,8138-8142$.

36 M. Anwar, S. Kumar, F. Ahmed, G. Kim and B. H. Koo, Journal of nanoscience and nanotechnology, 2012, 12, 5523-5526.

37 Y. Dang-Hyok, K. Raju, M. Bong-Ki and P. V. Reddy, Ceram. Int., 2014, 40, 13497-13505.

38 J. Moradi, M. Ghazi, M. Ehsani and P. Kameli, Journal of Solid State Chemistry, 2014, 215, 1-7.

39 A. R. Von Hippel, Dielectric materials and applications, Artech House, London, United Kingdom, 1954

40 K. C. Kao, Dielectric phenomena in solids, Elsevier Academic press, Amsterdam, 2004.

41 P. M. Gross Jr and R. C. Taylor, J. Am. Chem. Soc., 1950, 72, 20752080.

42 G. Korotchenkov, in Handbook of Gas Sensor Materials: Properties, Advantages and Shortcomings for Applications Volume 1: Conventional Approaches, Springer New York, USA, 2013, p. 93-94.

43 H. Frederikse, Handbook of Chemistry and Physics, 1998, 1999, 12 48.

44 K. U. Madhu and C. K. Mahadevan, International Journal of Engineering Research and Applications, 2013, 3, 2264-2267.

45 C. O. Kappe, B. Pieber and D. Dallinger, Angewandte Chemie International Edition, 2013, 52, 1088-1094.
46 M. A. Herrero, J. M. Kremsner and C. O. Kappe, J. Org. Chem., 2008, 73, 36-47.

47 C. R. Strauss, Angewandte Chemie International Edition, 2002, 41, 3589-3591.

48 M. Nüchter, B. Ondruschka, W. Bonrath and A. Gum, Green Chem., 2004, 6, 128-141.

49 G. Tompsett, W. Conner and K. Yngvesson, ChemPhysChem, 2006, 7, 296-319.

50 B. L. Hayes, Aldrichimica Acta, 2004, 37, 66-77.

51 R. Roy, R. Peelamedu, L. Hurtt, J. Cheng and D. Agrawal, Materials Research Innovations, 2002, 6, 128-140.

52 J. Giri, T. Sriharsha, S. Asthana, T. K. G. Rao, A. K. Nigam and D. and Bahadur, J Magn Magn Mater, 2005, 293, 55-61.

53 S. Farhadi, Z. Momeni and M. Taherimehr, J. Alloys Compounds, 2009, 471, L5-L8.

54 S. D. Hutagalung, M. I. M. Ibrahim and Z. A. Ahmad, Ceram. Int., 2008, 34, 939-942.

55 W. Sun, C. Li, J. Li and W. Liu, Mater. Chem. Phys., 2006, 97, 481487.

56 H. Yu, H. Liu, D. Luo and M. Cao, J. Mater. Process. Technol., 2008, 208, 145-148.

57 K. McBride, N. Partridge, S. Bennington-Gray, S. Felton, L. Stella and D. Poulidi, Mater. Res. Bull., 2017, 88, 69-77.

58 K. Momma and F. Izumi, Journal of Applied Crystallography, 2011, 44, 1272-1276.

59 R. R. Wildeboer, P. Southern and Q. A. and Pankhurst, J. Phys. D, 2014, 47, 495003-14pp.

60 C. T. Kniess, J. C. de Lima and P. B. Prates, in Sintering - Methods and Products, ed. V. Shatokha, INTECH, Open Access Publisher, 2012, p. 293-318.

61 J. Binner, N. Hassine and T. Cross, J. Mater. Sci., 1995, 30, 53895393.

62 N. Zhang, W. Yang, W. Ding, D. Xing and Y. Du, Solid State Commun., 1999, 109, 537-542.

63 T. Degen, M. Sadki, E. Bron, U. König and G. Nénert, Powder Diffraction, 2014, 29, S13-S18.

64 V. K. Pecharsky and P. Y. Zavalij, in Fundamentals of powder diffraction and structural characterization of materials, Springer, New York, USA, 2009, p. 347-405.

65 A. J. Blake and W. Clegg, Crystal structure analysis: principles and practice, Oxford University Press, Oxford, United Kingdom, 2009.

66 A. Millis, Physical Review B, 1996, 53, 8434-8441.

67 J. Zhou and J. B. and Goodenough, Physical Review B, 2001, 64, 024421.

68 A. Millis, B. I. Shraiman and R. Mueller, Phys. Rev. Lett., 1996, 77, 175-179.

69 R. Hong, T. Pan and H. Li, J Magn Magn Mater, 2006, 303, 60-68. 70 D. R. Sahu, B. K. Roul, P. Pramanik and J. Huang, Physica B: Condensed Matter, 2005, 369, 209-214.

71 P. Kameli, H. Salamati and A. Aezami, J. Appl. Phys., 2006, 100, 053914-4 pp.

72 L. Balcells, J. Fontcuberta, B. Martinez and X. Obradors, Physical Review B, 1998, 58, R14697-R14700.

73 D. H. Manh, P. T. Phong, P. H. Nam, D. K. Tung, N. X. Phuc and I. Lee, Physica B: Condensed Matter, 2014, 444, 94-102.

74 M. Kačenka, O. Kaman, Z. Jirák, M. Maryško, P. Žvátora, S. Vratislav and I. Lukeš, J. Appl. Phys., 2014, 115, $17 \mathrm{~B} 525$.

75 A. Rostamnejadi, H. Salamati, P. Kameli and H. Ahmadvand, J Magn Magn Mater, 2009, 321, 3126-3131.

76 A. M. Tishin and Y. I. and Spichkin, in The magnetocaloric effect and its applications, CRC Press, Florida, U.S., 2003, p. 4-62. 